\title{
INVERSE SEMI-BRACES AND THE YANG-BAXTER EQUATION
}

\author{
Francesco Catino \\ Dipartimento di Matematica e Fisica \\ "Ennio De Giorgi" \\ Università del Salento \\ Via Provinciale Lecce-Arnesano \\ 73100 Lecce (Italy) \\ francesco.catino@unisalento.it
}

\author{
Marzia Mazzotta \\ Dipartimento di Matematica e Fisica \\ "Ennio De Giorgi" \\ Università del Salento \\ Via Provinciale Lecce-Arnesano \\ 73100 Lecce (Italy) \\ marzia.mazzotta@unisalento.it
}

\author{
Paola Stefanelli \\ Dipartimento di Matematica e Fisica \\ "Ennio De Giorgi" \\ Università del Salento \\ Via Provinciale Lecce-Arnesano \\ 73100 Lecce (Italy) \\ paola.stefanelli@unisalento.it
}

\begin{abstract}
The main aim of this paper is to provide set-theoretical solutions of the Yang-Baxter equation that are not necessarily bijective, among these new idempotent ones. In the specific, we draw on both to the classical theory of inverse semigroups and to that of the most recently studied braces, to give a new research perspective to the open problem of finding solutions. Namely, we have recourse to a new structure, the inverse semi-brace, that is a triple $(S,+, \cdot)$ with $(S,+)$ a semigroup and $(S, \cdot)$ an inverse semigroup satisfying the relation $a(b+c)=a b+a\left(a^{-1}+c\right)$, for all $a, b, c \in S$, where $a^{-1}$ is the inverse of $a$ in $(S, \cdot)$. In particular, we give several constructions of inverse semi-braces which allow for obtaining solutions that are different from those until known.
\end{abstract}

Keywords Quantum Yang-Baxter equation · set-theoretical solution · inverse semigroups · brace · semi-brace · skew brace $\cdot$ asymmetric product

$\begin{array}{llllll}\text { MSC } 2020 & 16 \mathrm{~T} 25 & 81 \mathrm{R} 50 & 16 \mathrm{Y} 99 & 16 \mathrm{~N} 20 & 20 \mathrm{M} 18\end{array}$

\section{Introduction}

The quantum Yang-Baxter equation first appeared in theoretical physics in a paper by Yang [57] to study a onedimensional quantum mechanical many body problem. In an independent way, Baxter [4] solved an eight-vertex model in statistical mechanics by means of this equation. In subsequent years, the interest in such equation has vastly increased: it led to the foundations of the theory of quantum groups and it also appeared in topology and algebra above all for its connections with braid groups and Hopf algebras. In the '90s, Drinfel'd [27] posed the question of finding all the so called set-theoretical solutions of the Yang-Baxter equation. Specifically, given a set $S$, a map $r: S \times S \longrightarrow S \times S$ satisfying the relation

$$
\left(r \times \operatorname{id}_{S}\right)\left(\operatorname{id}_{S} \times r\right)\left(r \times \operatorname{id}_{S}\right)=\left(\operatorname{id}_{S} \times r\right)\left(r \times \operatorname{id}_{S}\right)\left(\operatorname{id}_{S} \times r\right)
$$


is said to be a set-theoretical solution of the Yang-Baxter equation, or briefly a solution. The map $r$ is usually written as $r(x, y)=\left(\lambda_{x}(y), \rho_{y}(x)\right)$, with $\lambda_{x}$ and $\rho_{y}$ maps from $S$ into itself, for all $x, y \in S$. One says that a solution $r$ is left non-degenerate if $\lambda_{x}$ is bijective, for every $x \in S$, right non-degenerate if $\rho_{y}$ is bijective, for every $y \in S$, and non-degenerate if $r$ is both left and right non-degenerate. If $r$ is neither left nor right non-degenerate, then it is called degenerate. Determining all the solutions is still an open problem and it has drawn the attention of several mathematicians. A large number of works related to this topic has been produced in recent years, actually. The milestones are the papers by Etingof, Schedler and Soloviev [28], Gateva-Ivanova and Van den Bergh [30], Lu, Yan, and Zhu [38], and Soloviev [53], where a greater attention has been posed on non-degenerate bijective solutions. Subsequently, involutive solutions have been profusely investigated by many authors, as mostly illustrated into details in the introduction of the paper by Cedó, Jespers, and Okniński [21]. The most used approach is based on left braces, algebraic structures introduced by Rump [47] that include the Jacobson radical rings. In particular, such structures are involved for obtaining non-degenerate solutions which are also involutive, i.e., $r^{2}=\mathrm{id}$. In this way, Rump traced a novel research direction and later fruitful results on these kind of solutions appeared, as one can see in the survey by Cedó [19], along the references therein. To classify involutive solutions, Rump [46] also involved another algebraic structure, that is the left cycle set. Interesting contributions in this framework have been obtained, for example, see [48, 55, 7, 6, 8, 9, 11, 51].

Bijective solutions, not necessarily involutive, can be produced through skew left braces, algebraic structures introduced by Guarnieri and Vendramin [31]. Also in this case several works can be found, for instance [52, 14, 41, 24, 34, 49, 2, 1, 5]. Note that skew left braces always produce solutions that are non-degenerate [31, Theorem 3.1] and, in the finite case, they are such that $r^{2 n}=\mathrm{id}$, as shown by Smoktunowicz and Vendramin [52, Theorem 4.13]. First instances of bijective solutions which are degenerate was found by Yang [58, Theorem 4.15], who studied the interplay between $k$-graphs and the Yang-Baxter equation.

Recently, the focus has been gradually shifted to solutions that are not necessarily bijective. Among these solutions, the most studied are idempotent ones. The investigation of such maps has been mainly started by Lebed [37], who provided a series of examples related to free and free commutative monoids, to factorizable monoids, and to distributive lattices. Later, Matsumoto and Shimizu [40] also approached to idempotent solutions of dynamical type in a categorical framework. Moreover, Stanovskỳ and Vojtěchovskỳ [54] dealt with idempotent left non-degenerate solutions which are in one-to-one correspondence with twisted Ward left quasigroups and, in particular, they enumerated those that are latin and idempotent. In addition, we mention Cvetko-Vah and Verwimp [26] who gave new examples of degenerate solutions including the idempotent ones, using the algebraic tool of the skew lattice.

More in general, Catino, Colazzo, and Stefanelli [13] showed that the algebraic structure of left semi-brace turns out to be a useful tool for producing left non-degenerate solutions which are not bijective. Under mild assumptions, Jespers and Van Antwerpen [35] determined soon after degenerate solutions through a slight generalization of left semi-braces. Furthermore, construction techniques that allow for providing new solutions that are both not necessarily non-degenerate and bijective starting from given ones have been introduced. Specifically, in [16] it is displayed how to find solutions of finite order by introducing the technique called the matched product of solutions inspired to the 
matched product of semi-braces [15]. Another way to determine solutions of finite order which are non-bijective, even starting from bijective ones, is the strong semilattice of solutions, contained in [17], basing on the strong semilattice of semigroups. Concretely, instances of such solutions are obtained involving the structure of generalized semi-brace. Catino, Mazzotta, and Stefanelli [18] also provided new degenerate solutions by means of a technique which involves solutions to the pentagon equation, another basic equation of mathematical physics. Newly, Cedó, Jespers, and Verwimp [23] investigated the structure monoid of arbitrary solutions not necessarily bijective, inspired by the work of Gateva-Ivanova and Majid [29]. Finally, Castelli, Catino, and Stefanelli [10] developed a theory of extensions for left non-degenerate solutions involving the algebraic structure of $q$-cycle set.

In this paper, we introduce a new algebraic structure, namely the left inverse semi-brace, which turns out to be a useful tool for determining solutions not necessarily bijective. In particular, this notion involves inverse semigroups, which we recall to be semigroups $S$ such that, for each $x \in S$, there exists a unique $x^{-1} \in S$ satisfying $x x^{-1} x=x$ and $x^{-1} x x^{-1}=x^{-1}$. Inverse semigroup theory was initiated in the 1950 s and it has been extensively studied during the years. Most of the known results up to the early 1980s are summarized into the monograph by Petrich [43]. Many works have been appeared on inverse semigroups until now and whole chapters of classical semigroups books have been dedicated to this topic, like that by Howie [33]. One can draw on to the large and currently investigated theory of inverse semigroups to give a new research perspective to the open problem of finding solutions.

A triple $(S,+, \cdot)$ is said to be a left inverse semi-brace if $(S,+)$ is a semigroup, $(S, \cdot)$ an inverse semigroup, and it holds

$$
a(b+c)=a b+a\left(a^{-1}+c\right)
$$

for all $a, b, c \in S$. This structure includes left semi-braces, introduced by Catino, Colazzo, and Stefanelli in [13] and Jespers and Van Antwerpen in [35], where the semigroup $(S, \cdot)$ is a group, and generalized semi-braces treated by the first authors in [17] with $(S, \cdot)$ a Clifford semigroup. If $(S,+, \cdot)$ is a left semi-brace with $(S,+)$ a left cancellative semigroup, then the map associated to $S$, i.e., the map $r_{S}: S \times S \rightarrow S \times S$ given by

$$
r_{S}(a, b)=\left(a\left(a^{-1}+b\right),\left(a^{-1}+b\right)^{-1} b\right)
$$

for all $a, b \in S$, is always a solution that is also left non-degenerate [13]. Instead, if $S$ is an arbitrary left semi-brace, the map $r_{S}$ is not a solution in general and, in this context, a characterization has been given in [17, Theorem 3]. In light of this, we provide sufficient conditions in order that the map $r_{S}$ associated to an inverse semi-brace $S$ is a solution.

Our attention is turned to the study of inverse semi-braces $S$ for which the map $r_{S}$ is a solution. Specifically, we show how these structures produce a variety of new solutions which are non-bijective and degenerate. Just to give an idea, fixed a right zero semigroup $(S,+)$, if $(S, \cdot)$ is an arbitrary inverse semigroup, then the map $r_{S}$ associated to the left inverse semi-brace $(S,+, \cdot)$, that is given by $r_{S}(a, b)=\left(a b, b^{-1} b\right)$, is an idempotent and two-sided degenerate solution. Moreover, there are as many solutions $r_{S}$ as there are inverse semigroups $(S, \cdot)$. Thus, the number of finite 
inverse semigroups allows for determining a lower bound for finite idempotent solutions of the form of $r_{S}$. In this respect, we refer the reader to [39], where it has been provided an algorithm for the enumeration of inverse semigroups of finite order. We highlight that if the semigroup $(S, \cdot)$ is completely regular, hence $(S,+, \cdot)$ is a generalized semibrace, such a map $r_{S}$ is not a solution, in general.

The article is structured as it follows. The first two sections are devoted to introducing left inverse semi-braces and some easy examples. Although the description of the semigroup $(S,+)$ of a left inverse semi-brace $(S,+, \cdot)$ is rather complicated, we show that in the specific case of $S$ a left semi-brace, $(S,+)$ is a rectangular semigroup. Furthermore, we focus on left inverse semi-braces which give solutions. Specifically, we find sufficient conditions to obtain them and show that, if $S$ is a left semi-brace, then the condition provided in the characterization [17, Theorem 3] is satisfied. In the remainder of the work, given two left inverse semi-braces $S$ and $T$, we present some constructions of left inverse semi-braces having the Cartesian Product $S \times T$ as underlying set. Into detail, in the third section we extend the matched product of left semi-braces, contained in [15], to the matched product of left inverse semi-braces. Moreover, we demonstrate that also in this case any matched product of $S$ and $T$ for which the maps $r_{S}$ and $r_{T}$ are solutions gives rise to a new solution that is exactly the matched product of $r_{S}$ and $r_{T}$. As an application, we show that already the easier case of the semidirect product leads to find various and new examples of solutions, of which we determine their order.

A new construction of left inverse semi-braces, including the semidirect product of left inverse semi-braces is introduced in the fourth section and we call it the double semidirect product. Assuming that the maps $r_{S}$ and $r_{T}$ associated to $S$ and $T$, respectively, are solutions, we aim to provide a lot of examples of solutions of various kinds associated to the double semi-direct product of $S$ and $T$, not only in the specific class of the degenerate and non-bijective ones. In particular, if $S$ and $T$ are arbitrary left semi-braces or skew left braces, we show that easy and usable conditions allow for obtaining a new solution on $S \times T$.

In the last section, we extend the asymmetric product of left cancellative left semi-braces given in [13] to left inverse semi-braces. Let us note that the asymmetric product of left braces proved useful to obtain rather systematic constructions of regular subgroups of the affine group [12], to investigate simple left braces [3, 20, 22], and it has been related to another construction of finite braces, the upper shifted semi-direct product of braces, in a recent work [50]. To our purpose, we need the notion of $\delta$-cocycle on semigroups, inspired to that used for groups (see the Schreier's extension in [32, Theorem 15.1.1]). Let us observe that this is not a simple readjustment of the definition introduced in [13], since it involves entirely the additive structures of the left inverse semi-braces. We privilege again left inverse semi-braces having solutions and we provide sufficient conditions to obtain solutions in the case of arbitrary left semi-braces.

\section{Left inverse semi-braces: definitions and examples}

In this section, we introduce the definition of left inverse semi-brace and we give some basic examples. Further and more challenging examples will be presented later. 
For the ease of the reader, we initially recall essential notions on inverse semigroups for our treatment. For further details one can see the book by Howie [33] or the monograph by Petrich [43]. A semigroup $S$ is called an inverse semigroup if, for each $a \in S$, there exists a unique element $a^{-1}$ of $S$ such that $a a^{-1} a=a$ and $a^{-1} a a^{-1}=a^{-1}$. We call such an element $a^{-1}$ the inverse of $a$.

Evidently, every group is an inverse semigroup. The behaviour of inverse elements in an inverse semigroup is similar to that in a group, as we recall below.

Lemma 1. Given an inverse semigroup $S$, they hold $(a b)^{-1}=b^{-1} a^{-1}$ and $\left(a^{-1}\right)^{-1}=a$, for all $a, b \in S$.

Lemma 2. If $S$ and $T$ are inverse semigroups and $\Phi$ is a homomorphism from $S$ into $T$, then $\Phi\left(a^{-1}\right)=\Phi(a)^{-1}$, for any $a \in S$.

Note that if $a$ is an element of an inverse semigroup $S$, then $a a^{-1}$ and $a^{-1} a$ are idempontent elements of $S$. Moreover, the set $\mathrm{E}(S)$ of the idempotents is a commutative subsemigroup of $S$ and $e=e^{-1}$, for every $e \in \mathrm{E}(S)$.

Finally, we recall that an inverse semigroup in which its idempotent elements are central is called a Clifford semigroup.

Now, we give the notion of left inverse semi-brace.

Definition 3. Let $S$ be a set with two operations + and $\cdot$ such that $(S,+)$ is a semigroup (not necessarily commutative) and $(S, \cdot)$ is an inverse semigroup. Then, we say that $(S,+, \cdot)$ is a left inverse semi-brace if

$$
a(b+c)=a b+a\left(a^{-1}+c\right)
$$

holds, for all $a, b, c \in S$. We call $(S,+)$ and $(S, \cdot)$ the additive semigroup and the multiplicative semigroup of $S$, respectively.

A right inverse semi-brace is defined similarly, by replacing condition (1) with $(a+b) c=\left(a+c^{-1}\right) c+b c$, for all $a, b, c \in S$.

A two-sided inverse semi-brace $(S,+, \cdot)$ is a left inverse semi-brace that is also a right inverse semi-brace with respect to the same operations + and $\cdot$

Clearly, any left semi-brace is a left inverse semi-brace, since in this case $(S, \cdot)$ is a group. Other examples of left inverse semi-braces are generalized left semi-braces $(S,+, \cdot)$, introduced in [17], with $(S, \cdot)$ a Clifford semigroup. For the ease of the reader, we recall that $(S,+, \cdot)$ is a generalized semi-brace if $(S,+)$ is a semigroup, $(S, \cdot)$ is a completely regular semigroup and condition (1) is satisfied.

Any arbitrary inverse semigroup gives easily rise to left inverse semi-braces, as we will show in the next examples.

Example 1. If $(S, \cdot)$ is an inverse semigroup and $(S,+)$ is a right zero semigroup or a left zero semigroup, then $S$ is an inverse two-sided semi-brace, which we call trivial inverse semi-brace. Clearly, if $|S|>1$, then such trivial semi-braces are not isomorphic. 
Example 2. Let $(S, \cdot)$ be an inverse semigroup and set $a+b=a a^{-1} b$, for all $a, b \in S$. Then, $S$ is a left inverse semi-brace. Note that if $(S, \cdot)$ is a Clifford semigroup, then $S$ is a two-sided inverse semi-brace. Similarly, the same is true if we consider the opposite sum, i.e., $a+b=b b^{-1} a$, for all $a, b \in S$.

Example 3. Let $(S, \cdot)$ be an inverse semigroup, $e \in \mathrm{E}(S)$ and set $a+b=b e$, for all $a, b \in S$. Then, it is easy to check that $S$ is a left inverse semi-brace. Note that, if $e$ is central, then $(S,+. \cdot)$ is also a right inverse semi-brace.

The following are examples of left inverse semi-braces obtained starting from an arbitrary Clifford semigroup.

Examples 4. Let $(S, \cdot)$ be a Clifford semigroup. If $a+b=a b$, for all $a, b \in S$, then $S$ is a generalized two-sided semi-brace (see [17, Example 9]). The same is true if we take the opposite sum, i.e., $a+b=b a$, for all $a, b \in S$.

Now, we present a construction of left inverse semi-braces which involves strong semilattice of inverse semigroups, that it is well-known to be inverse (see [43, Ex.(ii), p.90]). Note that a similar construction has already been considered in the case of generalized left semi-braces in [17, Proposition 10]. For this reason, we omit the detailed proof.

Proposition 4. Let $Y$ be a (lower) semilattice, $\left\{S_{\alpha} \mid \alpha \in S\right\}$ a family of disjoint left inverse semi-braces. For each pair $\alpha, \beta$ of elements of $Y$ such that $\alpha \geq \beta$, let $\phi_{\alpha, \beta}: S_{\alpha} \rightarrow S_{\beta}$ be a homomorphism of left inverse semi-braces such that

1. $\phi_{\alpha, \alpha}$ is the identical automorphism of $S_{\alpha}$, for every $\alpha \in Y$,

2. $\phi_{\beta, \gamma} \phi_{\alpha, \beta}=\phi_{\alpha, \gamma}$, for all $\alpha, \beta, \gamma \in S$ such that $\alpha \geq \beta \geq \gamma$.

Then, $S=\bigcup\left\{S_{\alpha} \mid \alpha \in Y\right\}$ endowed by the addition and the multiplication defined by

$$
\begin{aligned}
a+b & :=\phi_{\alpha, \alpha \beta}(a)+\phi_{\beta, \alpha \beta}(b), \\
a b & :=\phi_{\alpha, \alpha \beta}(a) \phi_{\beta, \alpha \beta}(b),
\end{aligned}
$$

for every $a \in S_{\alpha}$ and $b \in S_{\beta}$, is a left inverse semi-brace. Such a left inverse semi-brace is said to be the strong semilattice $S$ of left inverse semi-brace $S_{\alpha}$ and is denoted by $S=\left[Y ; S_{\alpha}, \phi_{\alpha, \beta}\right]$.

The previous examples and Proposition 4 suggest that the structure of additive semigroup of a left inverse semibrace is rather complicated. At present, only partial results are known for left semi-braces. Under mild assumptions, for instance in the finite case, it has been proved that the additive semigroup $(S,+)$ of a left semi-brace $S$ is a completely simple semigroup (see [35, Theorem 2.8]). In more detail, $(S,+)$ is a rectangular group, i.e., it is isomorphic to the direct product of a group and a rectangular band, see [17, Theorem 3].

Now, we show that the additive semigroup of an arbitrary left semi-brace is a rectangular semigroup. In this regard, we recall that a semigroup $(S,+)$ is stationary on the right if

$$
a+b=a+c \Longrightarrow x+b=x+c
$$


holds, for all $a, b, c, x \in S$. By [25, Ex. 7, p. 98], any semigroup which is stationary on the right is rectangular. We recall that a semigroup $(S,+)$ is rectangular if it holds

$$
a+x=b+x=a+y \Longrightarrow a+x=b+y
$$

for all $a, b, x, y \in S$ (see [44, Definition III.5.14]). In addition, we recall that if $S$ is a left semi-brace, the identity 1 of the group $(S, \cdot)$ satisfies two special properties, namely, 1 is an idempotent element of $(S,+)$ and it is also a middle unit, i.e., $a+1+b=a+b$, for all $a, b \in S$ (see Lemma 2.4 (1) and Lemma 2.6 (ii) of [35]).

Proposition 5. Let $(S,+, \cdot)$ be a left semi-brace. Then, the semigroup $(S,+)$ is stationary on the right. Consequently, $(S,+)$ is a rectangular semigroup and they hold:

1. $E(S)$ is a rectangular band;

2. $e \in E(S)$ if and only if e is a middle unit.

Proof. Let $a, b, c \in S$ such that $a+b=a+c$. Then, we obtain

$$
\begin{aligned}
a+b=a+c & \Longrightarrow a\left(a^{-1}+a^{-1}(a+b)\right)=a\left(a^{-1}+a^{-1}(a+c)\right) \\
& \Longrightarrow 1+b=1+c .
\end{aligned}
$$

Hence, since 1 is a middle unit, we have that $x+b=x+1+b=x+1+c=x+c$, for every $x \in S$. Thus, the semigroup $(S,+)$ is stationary on the right and so it is rectangular.

Now, by [25, Ex. 7, p. 98] the set of idempotents $\mathrm{E}(S)$ is a rectangular band and any idempotent is a middle unit. Vice versa, [17, Proposition 4] completes the proof.

Finally, we note that, under specific assumptions on the additive structure, a left inverse semi-brace $(S,+, \cdot)$ is necessarily a skew left brace, as shown below. In this respect, we recall that if $(S,+)$ is left cancellative, then 1 is also a left identity (see [13, p. 165]).

Remark 1. Let $(S,+, \cdot)$ be a left inverse semi-brace. If $(S,+)$ is a left cancellative semigroup with a right identity 1 , then $(S, \cdot)$ is a group. Indeed, if $a \in S$, then

$$
a 1+1=1=a(1+1)=a 1+a\left(a^{-1}+1\right)=a 1+a a^{-1},
$$

hence $a a^{-1}=1$. Therefore, since every idempotent of $S$ can be expressed in the form $x x^{-1}$, with $x \in S$, we obtain that 1 is the unique idempotent in $(S, \cdot)$. Therefore, $S$ is a left cancellative left semi-brace. Since, in this case 1 is a left identity in $(S,+)$, it follows that $(S,+)$ is a monoid. As observed in [13, p. 167], the structure $S$ is necessarily a skew left brace. 


\section{Solutions associated to left inverse semi-braces}

In this section, we deal with solutions associated to left inverse semi-braces and we provide sufficient conditions to obtain them. In this way, we give several solutions which are associated to the examples in the previous section.

As is common in the semi-brace theory, given a left inverse semi-brace $(S,+, \cdot)$, let us consider the two maps $\lambda: S \rightarrow \operatorname{End}(S,+), a \mapsto \lambda_{a}$ from $S$ into the endomorphism semigroup of $(S,+)$ and $\rho: S \rightarrow S^{S}, b \mapsto \rho_{b}$ from $S$ into the semigroup of the maps from $S$ into itself defined by

$$
\lambda_{a}(b)=a\left(a^{-1}+b\right) \quad \rho_{b}(a)=\left(a^{-1}+b\right)^{-1} b,
$$

for all $a, b \in S$, respectively. Moreover, if $a, b, c \in S$, then $\lambda_{a b}(c)=a b b^{-1} a^{-1}+\lambda_{a} \lambda_{b}(c)$, where we observe that $a b b^{-1} a^{-1} \in \mathrm{E}(S)$. We call the map $r_{S}: S \times S \rightarrow S \times S$ given by

$$
r_{S}(a, b)=\left(\lambda_{a}(b), \rho_{b}(a)\right)
$$

for all $(a, b) \in S \times S$, the map associated to the left inverse semi-brace $(S,+, \cdot)$. Note that, if $(S,+, \cdot)$ is a left semibrace with $(S,+)$ a left cancellative semigroup, then the map $r_{S}$ is a left non-degenerate solution (see [13, Theorem 9]). Let us recall that not every left semi-brace gives rise to solutions, yet. In this context, in [16] a characterization has been provided.

Theorem 6 (Theorem 3, [16]). Let $(S,+, \cdot)$ be a left semi-brace. The map $r_{S}$ associated to $S$ is a solution if and only if

$$
a+\lambda_{b}(c)\left(1+\rho_{c}(b)\right)=a+b(1+c)
$$

holds, for all $a, b, c \in S$.

Observe that, as shown in [17, p. 8], if the map $\rho$ is an anti-homomorphism from the group $(S, \cdot)$ into the monoid of the maps from $S$ into itself, then the map $r_{S}$ associated to the left semi-brace $(S,+, \cdot)$ satisfies condition (2). In particular, if $(S,+)$ is a left cancellative semigroup, the condition (2) is satisfied, too.

In the following, we provide sufficient conditions to obtain solutions through left inverse semi-braces.

Theorem 7. Let $(S,+, \cdot)$ be a left inverse semi-brace and $r_{S}$ the map associated to $S$. If the following are satisfied

$$
\begin{aligned}
& \text { 1. }(a+b)(a+b)^{-1}(a+b c)=a+b c \\
& \text { 2. } \lambda_{a}(b)^{-1}+\lambda_{\rho_{b}(a)}(c)=\lambda_{a}(b)^{-1}+\lambda_{\left(a^{-1}+b\right)^{-1}} \lambda_{b}(c) \\
& \text { 3. } \rho_{b}(a)^{-1}+c=\left(b^{-1}+c\right)\left(\rho_{\lambda_{b}(c)}(a)^{-1}+\rho_{c}(b)\right),
\end{aligned}
$$

for all $a, b, c \in S$, then the map $r_{S}$ is a solution. 
Proof. It is a routine computation to verify that the map $r$ associated to $S$ given by $r(a, b)=\left(\lambda_{a}(b), \rho_{b}(a)\right)$ is a solution if and only if they hold

$$
\begin{aligned}
& \lambda_{a} \lambda_{b}(c)=\lambda_{\lambda_{a}(b)} \lambda_{\rho_{b}(a)}(c) \\
& \lambda_{\rho_{\lambda_{b}(c)}(a)} \rho_{c}(b)=\rho_{\lambda_{\rho_{b}(a)}(c)} \lambda_{a}(b) \\
& \rho_{c} \rho_{b}(a)=\rho_{\rho_{c}(b)} \rho_{\lambda_{b}(c)}(a)
\end{aligned}
$$

for all $a, b, c \in S$. Thus, if $a, b, c \in S$, we have that

$$
\begin{aligned}
\lambda_{\lambda_{a}(b)} \lambda_{\rho_{b}(a)}(c) & =\lambda_{a}(b)\left(\lambda_{a}(b)^{-1}+\lambda_{\rho_{b}(a)}(c)\right) \\
& =\lambda_{a}(b)\left(\lambda_{a}(b)^{-1}+\lambda_{\left(a^{-1}+b\right)^{-1}} \lambda_{b}(c)\right) \\
& =\lambda_{a}(b)\left(\left(a^{-1}+b\right)^{-1} a^{-1}+\left(a^{-1}+b\right)^{-1}\left(a^{-1}+b+\lambda_{b}(c)\right)\right) \\
& =\lambda_{a}(b)\left(a^{-1}+b\right)^{-1}\left(a^{-1}+\lambda_{b}(c)\right) \\
& =a\left(a^{-1}+b\right)\left(a^{-1}+b\right)^{-1}\left(a^{-1}+b\left(b^{-1}+c\right)\right) \\
& =a\left(a^{-1}+b\left(b^{-1}+c\right)\right) \\
& =a\left(a^{-1}+\lambda_{b}(c)\right)=\lambda_{a} \lambda_{b}(c) .
\end{aligned}
$$

\section{Moreover, we obtain}

$$
\begin{aligned}
\lambda_{\rho_{\lambda_{b}(c)}(a)} \rho_{c}(b) & =\rho_{\lambda_{b}(c)}(a)\left(\rho_{\lambda_{b}(c)}(a)^{-1}+\rho_{c}(b)\right) \\
& =\left(a^{-1}+\lambda_{b}(c)\right)^{-1} \lambda_{b}(c)\left(\rho_{\lambda_{b}(c)}(a)^{-1}+\rho_{c}(b)\right) \\
& =\left(a^{-1}+\lambda_{b}(c)\right)^{-1} b\left(b^{-1}+c\right)\left(\rho_{\lambda_{b}(c)}(a)^{-1}+\rho_{c}(b)\right) \\
& =\left(a^{-1}+\lambda_{b}(c)\right)^{-1} b\left(\rho_{b}(a)^{-1}+c\right) \\
& =\left(a^{-1}+\lambda_{b}(c)\right)^{-1}\left(a^{-1}+b\right)\left(a^{-1}+b\right)^{-1} b\left(\rho_{b}(a)^{-1}+c\right) \\
& =\left(\left(a^{-1}+b\right)^{-1}\left(a^{-1}+\lambda_{b}(c)\right)\right)^{-1} \rho_{b}(a)\left(\rho_{b}(a)^{-1}+c\right) \\
& =\left(\left(a^{-1}+b\right)^{-1} a^{-1}+\lambda_{\left.\left(a^{-1}+b\right)^{-1} \lambda_{b}(c)\right)^{-1} \lambda_{\rho_{b}(a)}(c)}\right. \\
& =\left(\lambda_{a}(b)^{-1}+\lambda_{\left.\left(a^{-1}+b\right)^{-1} \lambda_{b}(c)\right)^{-1} \lambda_{\rho_{b}(a)}(c)}^{\text {by 3. }}\right. \\
& =\left(\lambda_{a}(b)^{-1}+\lambda_{\rho_{b}(a)}(c)\right)^{-1} \lambda_{\rho_{b}(a)}(c) \\
& =\rho_{\lambda_{\rho_{b}(a)}(c)} \lambda_{a}(b) .
\end{aligned}
$$


Finally, we get

$$
\begin{aligned}
\rho_{c} \rho_{b}(a) & =\left(\rho_{b}(a)^{-1}+c\right)^{-1} c \\
& =\left(\left(b^{-1}+c\right)\left(\rho_{\lambda_{b}(c)}(a)^{-1}+\rho_{c}(b)\right)\right)^{-1} c \\
& =\left(\rho_{\lambda_{b}(c)}(a)^{-1}+\rho_{c}(b)\right)^{-1}\left(b^{-1}+c\right)^{-1} c \\
& =\left(\rho_{\lambda_{b}(c)}(a)^{-1}+\rho_{c}(b)\right)^{-1} \rho_{c}(b) \\
& =\rho_{\rho_{c}(b)} \rho_{\lambda_{b}(c)}(a) .
\end{aligned}
$$

Therefore, the map $r_{S}$ is a solution on the left inverse semi-brace $S$.

Remark 2. If $(S,+, \cdot)$ is a left semi-brace, then the condition 1. in Theorem 7 trivially holds. Moreover, since $\lambda$ is a homomorphism from $(S, \cdot)$ into End $(S,+)$, we have that condition 2 . is clearly satisfied. In addition, since in this case $(S, \cdot)$ is a group, we obtain that

$$
\begin{aligned}
\rho_{b}(a)^{-1}+c & =b^{-1} b\left(b^{-1}\left(a^{-1}+b\right)+c\right) \\
& =b^{-1}\left(a^{-1}+b+\lambda_{b}(c)\right)=b^{-1}(a+b(1+c))
\end{aligned}
$$

and

$$
\begin{aligned}
& \left(b^{-1}+c\right)\left(\rho_{\lambda_{b}(c)}(a)^{-1}+\rho_{c}(b)\right) \\
& =b^{-1} b\left(b^{-1}+c\right)\left(\lambda_{b}(c)^{-1}\left(a^{-1}+\lambda_{b}(c)\right)+\rho_{c}(b)\right) \\
& =b^{-1} \lambda_{b}(c)\left(\lambda_{b}(c)^{-1}\left(a^{-1}+\lambda_{b}(c)\right)+\rho_{c}(b)\right) \\
& =b^{-1}\left(a^{-1}+\lambda_{b}(c)+\lambda_{\lambda_{b}(c)} \rho_{b}(c)\right) \\
& =b^{-1}\left(a+\lambda_{b}(c)\left(1+\rho_{c}(b)\right)\right)
\end{aligned}
$$

hence the condition 3 . in Theorem 7 holds if and only if (2) in Theorem 6 is satisfied.

It is a routine computation to verify that all the examples of left inverse semi-braces provided until now satisfy the conditions of Theorem 7, hence the maps associated to each left inverse semi-brace are solutions. Below, we list these solutions and we highlight some properties about their behavior. In general, they are not bijective and lie in the class of degenerate ones.

Examples 5. Let $(S, \cdot)$ be an arbitrary inverse semigroup. If $S$ is the trivial left inverse semi-brace in Example 1 with $(S,+)$ a right zero semigroup, the map $r_{S}$ associated to $S$ given by

$$
r_{S}(a, b)=\left(a b, b^{-1} b\right)
$$


for all $a, b \in S$, is an idempotent solution. Similarly, if $S$ is the trivial left inverse semi-brace with $(S,+)$ a left zero semigroup, we get the idempotent solution

$$
r_{S}(a, b)=\left(a a^{-1}, a b\right)
$$

for all $a, b \in S$.

Note that if $|S|>1$ such solutions are not isomorphic in the sense of [21]. In addition, since they are strictly linked to a given inverse semigroup, it is clear that the number of such semigroups determines a lower bound for idempotent solutions. In this regard, we highlight that, recently, in [39] it has been provided an algorithm for the enumeration of the inverse semigroups of order $n$ (up to isomorphism). Furthermore, these maps are added to the class of idempotent solutions which are known until now [37, 40, 26, 15, 17].

Remark 3. Note that if $(S, \cdot)$ is a Clifford semigroup, the map $r(a, b)=\left(a b, b^{-1} b\right)$ associated to the trivial left inverse semi-brace in Examples[5, where the first component is exactly the multiplication in the semigroup $S$, is still a solution to the quantum Yang-Baxter equation, i.e., it satisfies the relation $r_{12} r_{13} r_{23}=r_{23} r_{13} r_{12}$, and to the pentagon equation, i.e., it holds $r_{23} r_{13} r_{12}=r_{12} r_{23}$. Thus, it belongs to the class of solutions of pentagonal type (for more details see [18, Proposition 8]). Similarly, recalling that a map $r$ is a solution if and only if $\tau r$ is a solution of the quantum Yang-Baxter equation, with $\tau$ the flip map on $S \times S$, then the map $\tau r(a, b)=\left(a b, a a^{-1}\right)$ is a solution of pentagonal type, too.

Examples 6. The map $r_{S}$ associated to the left inverse semi-braces $S$ in Example 2 with $a+b=a a^{-1} b$, for all $a, b \in S$, given by

$$
r_{S}(a, b)=\left(a b, a b(a b)^{-1}\right)
$$

for all $a, b \in S$, is an idempotent solution. Analogously, the map $t_{S}$ associated to the left inverse semi-braces $S$ in Example 2 with $a+b=a a^{-1} b$, for all $a, b \in S$, defined by

$$
t_{S}(a, b)=\left(a b(a b)^{-1}, a b\right)
$$

for all $a, b \in S$, is an idempotent solution. Let us note that $t_{S}=\tau r_{S}$, consequently these two solutions are not isomorphic.

Example 7. Let $S$ be the left inverse semi-brace in Example 3 where $a+b=b \cdot e$, with $e \in \operatorname{E}(S) \cap \zeta(S)$, then the map

$$
r_{S}(a, b)=\left(a b e, e b^{-1} b\right)
$$


for all $a, b \in S$, is an idempotent solution on $S$. Moreover, let us observe that the solution $t_{S}$ associated to $S$ considered as right inverse semi-brace is given by

$$
t_{S}(a, b)=\left(a\left(a+b^{-1}\right)^{-1},\left(a+b^{-1}\right) b\right)=\left(a b e, e b^{-1} b\right)=r_{S}(a, b)
$$

for all $a, b \in S$.

Examples 8. The map $r_{S}$ associated to the left inverse semi-brace $S$ in Examples 4 with $a+b=a b$, for all $a, b \in S$, given by

$$
r_{S}(a, b)=\left(a a^{-1} b, b^{-1} a b\right)
$$

for all $a, b \in S$, is a solution. If in addition the Clifford semigroup $(S, \cdot)$ is commutative, then $r_{S}$ is a cubic solution, i.e., $r_{S}^{3}=r_{S}$, see [17, p. 12]. Similarly, the solution associated to the left inverse semi-brace $S$ in Examples 4 with $a+b=b a$, for all $a, b \in S$, is given by

$$
r_{S}(a, b)=\left(a b a^{-1}, a b^{-1} b\right)
$$

for all $a, b \in S$, and also in this case $r_{S}$ is cubic if $(S, \cdot)$ is commutative.

As mentioned above, if $S=\left[Y ; S_{\alpha}, \phi_{\alpha, \beta}\right]$ is a strong semilattice of left inverse semi-braces for which every $S_{\alpha}$ has $r_{S_{\alpha}}$ as a solution, then the map associated to $S$ is a solution. This is a consequence of the technique named strong semilattice of solutions introduced in [17, Theorem 12].

Theorem 8. Let $S=\left[Y ; S_{\alpha}, \phi_{\alpha, \beta}\right]$ be a strong semilattice of left inverse semi-braces. If $S_{\alpha}$ has $r_{\alpha}$ as a solution, for every $\alpha \in Y$, then the map $r_{S}$ given by

$$
r_{S}(x, y):=r_{\alpha \beta}\left(\phi_{\alpha, \alpha \beta}(x), \phi_{\beta, \alpha \beta}(y)\right)
$$

for all $x \in S_{\alpha}$ and $y \in S_{\beta}$, is a solution on $S$.

In the remainder of this work, we provide constructions of left inverse semi-braces which allow to determine solutions starting from known left inverse semi-braces $S$ having $r_{S}$ as solution. In the specific, we will focus on constructions on the Cartesian product of left inverse semi-braces.

\section{The matched product of left inverse semi-braces}

This section is devoted to extend the construction of the matched product of left semi-braces contained in [15] to the class of the left inverse semi-braces. Moreover, we show that any matched product of two left inverse semi-braces $S$ and $T$ for which the maps $r_{S}$ and $r_{T}$ are solutions gives rise to a new solution that is exactly the matched product 
of $r_{S}$ and $r_{T}$. Finally, we provide various examples of solutions in the easier case, the semidirect product, and we highlight some properties about their order.

We begin by reminding the classical Zappa product of two semigroups contained in [36]. Let $S$ and $T$ be semigroups, $\sigma: T \rightarrow S^{S}$ and $\delta: S \rightarrow T^{T}$ maps, and set ${ }^{u} a:=\sigma(u)(a)$ and $u^{a}:=\delta(a)(u)$, for all $a \in S$ and $u \in T$. If the following conditions are satisfied

$$
\begin{aligned}
& { }^{u}(a b)={ }^{u} a{ }^{u^{a}} b \\
& { }^{u v} a={ }^{u}\left({ }^{v} a\right) \\
& (u v)^{a}=u^{v} a v^{a} \\
& u^{a b}=\left(u^{a}\right)^{b}
\end{aligned}
$$

for all $a, b \in S$ and $u, v \in T$, then $S \times T$ is a semigroup with respect to the operation defined by

$$
(a, u)(b, v)=\left(a^{u} b, u^{b} v\right)
$$

for all $a, b \in S$ and $u, v \in T$. In the following theorem, we give the necessary conditions for Zappa product of inverse semigroups to be inverse. Hereinafter, for the ease of the reader, we use the letters $a, b, c$ for $S$ and $u, v, w$ for $T$.

Theorem 9. (cf. [56, Theorem 4]) Let $S$ and $T$ be inverse semigroups, $\sigma: T \rightarrow S^{S}$ and $\delta: S \rightarrow T^{T}$ maps satisfying (Z1) and (Z2). If $\sigma(T) \subseteq \operatorname{Aut}(S)$ and $\delta(S) \subseteq \operatorname{Aut}(T)$ and

$$
a^{u} a=a, u^{a} u=u \Longrightarrow{ }^{u} a=a, u^{a}=u
$$

holds, for all $a \in S$ and $u \in T$, then $S \times T$ endowed with the operation (3) is an inverse semigroup.

Coherently with the notation of the matched product used in the context of left semi-braces in [17], we introduce suitable maps $\alpha$ and $\beta$ which allow for obtaining a new left inverse semi-brace having the multiplicative semigroup isomorphic to a Zappa product of the starting inverse semigroups.

Definition 10. Let $S$ and $T$ be left inverse semi-braces, $\alpha: T \rightarrow \operatorname{Aut}(S)$ a homomorphism of inverse semigroups from $(T, \cdot)$ into the automorphism group of $(S,+)$, and $\beta: S \rightarrow \operatorname{Aut}(T)$ a homomorphism of inverse semigroups from $(S, \cdot)$ into the automorphism group of $(T,+)$ such that

$$
\begin{gathered}
\alpha_{u}\left(\alpha_{u}^{-1}(a) b\right)=a \alpha_{\beta_{a}^{-1}(u)}(b) \quad \beta_{a}\left(\beta_{a}^{-1}(u) v\right)=u \beta_{\alpha_{u}^{-1}(a)}(v) \\
\alpha_{u}\left(\alpha_{u}^{-1}(a) a\right)=a, \beta_{a}\left(\beta_{a}^{-1}(u) u\right)=u \Longrightarrow \alpha_{u}(a)=a, \beta_{a}(u)=u
\end{gathered}
$$

hold, for all $a, b \in S$ and $u, v \in T$. Then, $(S, T, \alpha, \beta)$ is called a matched product system of left inverse semi-braces.

Remark 4. Note that if $e \in E(T)$, then $\alpha_{e}=\operatorname{id}_{S}$. In fact, there exists $u \in T$ such that $e=u u^{-1}$ and so

$$
\alpha_{e}(a)=\alpha_{u u^{-1}}(a)=\alpha_{u} \alpha_{u^{-1}}(a)=\alpha_{u} \alpha_{u}^{-1}(a)=a
$$


for every $a \in S$. Similarly, if $e \in \mathrm{E}(S)$, then $\beta_{e}=\operatorname{id}_{T}$.

Hereinafter, for every fixed pair $(a, u) \in S \times T$, we set $\bar{a}:=\alpha_{u}^{-1}(a)$ and $\bar{u}:=\beta_{a}^{-1}(u)$, whenever it is convenient. Now, we introduce a preparatory lemma.

Lemma 11. Let $(S, T, \alpha, \beta)$ be a matched product system of left inverse semi-braces. Then, the following

$$
\begin{aligned}
& \left(\alpha_{u}^{-1}(a)\right)^{-1}=\alpha_{\beta_{a}^{-1}(u)}^{-1}\left(a^{-1}\right) \\
& \left(\beta_{a}^{-1}(u)\right)^{-1}=\beta_{\alpha_{u}^{-1}(a)}^{-1}\left(u^{-1}\right)
\end{aligned}
$$

hold, for every $(a, u) \in S \times T$.

Proof. We prove the first statement, since the second one can be shown with the same computations where the roles of $\alpha$ and $\beta$ are reversed. If $(a, u) \in S \times T$, we have that

$$
\begin{aligned}
\alpha_{\bar{u}}^{-1}\left(a^{-1}\right) \bar{a} \alpha_{\bar{u}}^{-1}\left(a^{-1}\right) & =\alpha_{\bar{u}}^{-1}\left(a^{-1}\right) \alpha_{u}^{-1}\left(a \alpha_{\bar{u}} \alpha_{\bar{u}}^{-1}\left(a^{-1}\right)\right) \\
& =\alpha_{\bar{u}}^{-1}\left(a^{-1}\right) \alpha_{u}^{-1}\left(a a^{-1}\right) \\
& =\alpha_{\bar{u}}^{-1}\left(a^{-1} \alpha_{\beta_{a^{-1}}^{-1}(\bar{u})} \alpha_{u}^{-1}\left(a a^{-1}\right)\right) \\
& =\alpha_{\bar{u}}^{-1}\left(a^{-1} \alpha_{u} \alpha_{u}^{-1}\left(a a^{-1}\right)\right) \\
& =\alpha_{\bar{u}}^{-1}\left(a^{-1} a a^{-1}\right) \\
& =\alpha_{\bar{u}}^{-1}\left(a^{-1}\right) .
\end{aligned}
$$

Moreover, by Remark 4 , we have that

$$
\begin{aligned}
\bar{a} \alpha_{\bar{u}}^{-1}\left(a^{-1}\right) \bar{a} & =\alpha_{u}^{-1}\left(a \alpha_{\bar{u}} \alpha_{\bar{u}}^{-1}\left(a^{-1}\right)\right) \bar{a} \\
& =\alpha_{u}^{-1}\left(a a^{-1}\right) \bar{a} \\
& =\alpha_{u}^{-1}\left(a a^{-1} \alpha_{\beta_{a a^{-1}}^{-1}(u)} \alpha_{u}^{-1}(a)\right) \\
& =\alpha_{u}^{-1}\left(a a^{-1} \alpha_{u} \alpha_{u}^{-1}(a)\right) \\
& =\alpha_{u}^{-1}\left(a a^{-1} a\right) \\
& =\alpha_{u}^{-1}(a)=\bar{a} .
\end{aligned}
$$

Therefore, the condition (6) is proved.

Theorem 12. Let $(S, T, \alpha, \beta)$ be a matched product system of left inverse semi-braces. Then, $S \times T$ with respect to

$$
\begin{aligned}
(a, u)+(b, v) & :=(a+b, u+v) \\
(a, u)(b, v) & :=\left(\alpha_{u}\left(\alpha_{u}^{-1}(a) b\right), \beta_{a}\left(\beta_{a}^{-1}(u) v\right)\right),
\end{aligned}
$$


for all $(a, u),(b, v) \in S \times T$, is a left inverse semi-brace, called the matched product of $S$ and $T$ via $\alpha$ and $\beta$ and denoted by $S \bowtie T$.

Proof. Firstly, the structure $(S \times T,+)$ is trivially a semigroup. Moreover, $(S \times T, \cdot)$ is a semigroup, indeed, set $\sigma(u)(a)=\alpha_{u}(a)$ and $\delta(a)(u)=\beta_{\alpha_{u}(a)}^{-1}(u)$, for all $a \in S$ and $u \in T$, it follows that $(S \times T, \cdot)$ is a Zappa product (3) via $\sigma$ and $\delta$ and

$$
\varphi: S \times T \rightarrow S \times T,(a, u) \mapsto\left(a, \beta_{a}(u)\right)
$$

is trivially an isomorphism from the Zappa product of $S$ and $T$ into the semigroup $(S \times T, \cdot)$. To show that $(S \times T, \cdot)$ is inverse we prove that $(S \times T, \cdot)$ is regular and that its idempotents commute. If $(a, u) \in S \times T$, by Remark 4 , we obtain

$$
\begin{aligned}
& (a, u)\left(\alpha_{\bar{u}}^{-1}\left(a^{-1}\right), \beta_{\bar{a}}^{-1}\left(u^{-1}\right)\right)(a, u) \\
& =\left(a \alpha_{\bar{u}} \alpha_{\bar{u}}^{-1}\left(a^{-1}\right), u \beta_{\bar{a}} \beta_{\bar{a}}^{-1}\left(u^{-1}\right)\right)(a, u) \\
& =\left(a a^{-1}, u u^{-1}\right)(a, u) \\
& =\left(a a^{-1} \alpha_{\beta_{a a^{-1}}\left(u u^{-1}\right)}(a), u u^{-1} \beta_{\alpha_{u u^{-1}}^{-1}\left(a a^{-1}\right)}(u)\right) \\
& =\left(a a^{-1} \alpha_{u u^{-1}}(a), u u^{-1} \beta_{a a^{-1}}(u)\right)=\left(a a^{-1} a, u u^{-1} u\right)=(a, u)
\end{aligned}
$$

Furthermore,

$$
\begin{aligned}
& \left(\alpha_{\bar{u}}^{-1}\left(a^{-1}\right), \beta_{\bar{a}}^{-1}\left(u^{-1}\right)\right)(a, u)\left(\alpha_{\bar{u}}^{-1}\left(a^{-1}\right), \beta_{\bar{a}}^{-1}\left(u^{-1}\right)\right) \\
& =\left(\alpha_{\bar{u}}^{-1}\left(a^{-1}\right), \beta_{\bar{a}}^{-1}\left(u^{-1}\right)\right)\left(a \alpha_{\bar{u}} \alpha_{\bar{u}}^{-1}\left(a^{-1}\right), u \beta_{\bar{a}} \beta_{\bar{a}}^{-1}\left(u^{-1}\right)\right) \\
& =\left(\alpha_{\bar{u}}^{-1}\left(a^{-1}\right), \beta_{\bar{a}}^{-1}\left(u^{-1}\right)\right)\left(a a^{-1}, u u^{-1}\right) \\
& =\left(\alpha_{\bar{u}}^{-1}\left(a^{-1}\right) \alpha_{\beta_{\alpha_{\bar{u}}^{-1}\left(a^{-1}\right)}^{-1} \beta_{\bar{a}}^{-1}\left(u^{-1}\right)}\left(a a^{-1}\right), \beta_{\bar{a}}^{-1}\left(u^{-1}\right) \beta_{\alpha_{\beta_{\bar{a}}^{-1}\left(u^{-1}\right)} \alpha_{\bar{u}}^{-1}\left(a^{-1}\right)}\left(u u^{-1}\right)\right)
\end{aligned}
$$


Now, about the first component, we have that

$$
\begin{array}{ll}
\alpha_{\bar{u}}^{-1}\left(a^{-1}\right) \alpha_{\beta_{\alpha_{\bar{u}}^{-1}\left(a^{-1}\right)}^{-1} \beta_{\bar{a}}^{-1}\left(u^{-1}\right)}\left(a a^{-1}\right) & \text { by (7) } \\
=\alpha_{\bar{u}}^{-1}\left(a^{-1}\right) \alpha_{\beta_{\alpha_{\bar{u}}^{-1}\left(a^{-1}\right)}^{-1}}\left(\beta_{a}^{-1}(u)\right)^{-1}\left(a a^{-1}\right) & \text { by (7) } \\
=\alpha_{\bar{u}}^{-1}\left(a^{-1}\right) \alpha_{\left(\beta_{a^{-1}}^{-1} \beta_{a}^{-1}(u)\right)^{-1}}\left(a a^{-1}\right) & \text { by (4) } \\
=\alpha_{\bar{u}}^{-1}\left(a^{-1}\right) \alpha_{u}^{-1}\left(a a^{-1}\right) & \\
=\alpha_{\bar{u}}^{-1}\left(a^{-1} \alpha_{\beta_{a-1}^{-1} \beta_{a}^{-1}(u)} \alpha_{u}^{-1}\left(a a^{-1}\right)\right) & \\
=\alpha_{\bar{u}}^{-1}\left(a^{-1}\right)\left(a^{-1} \alpha_{u} \alpha_{u}^{-1}\left(a a^{-1}\right)\right) & \\
=\alpha_{\bar{u}}^{-1}\left(a^{-1} a a^{-1}\right) & \\
=\alpha_{\bar{u}}^{-1}\left(a^{-1}\right) &
\end{array}
$$

By reversing the role of $\alpha$ and $\beta$, we get that the second component is equal to $\beta_{\bar{a}}^{-1}\left(u^{-1}\right)$. Thus, $\left(\alpha_{\bar{u}}^{-1}\left(a^{-1}\right), \beta_{\bar{a}}^{-1}\left(u^{-1}\right)\right)$ in an inverse of $(a, u)$. Hence, $(S \times T, \cdot)$ is a regular semigroup. Now, by (5), note that $(a, u)$ is idempotent with respect to $\cdot$ if and only $a$ and $u$ are idempotent in $(S, \cdot)$ and $(T, \cdot)$, respectively. It follows that, if $(a, u)$ and $(b, v)$ are idempotents, then

$$
(a, u)(b, v)=\left(a \alpha_{\bar{u}}(b), u \beta_{\bar{a}}(v)\right)=\left(a \alpha_{u}(b), u \beta_{a}(v)\right)=(a b, u v)=(b a, v u)=\left(b \alpha_{\bar{v}}(a), v \beta_{\bar{b}}(u)\right)=(b, v)(a, u)
$$

Therefore, the idempotents commute and so $(S \times T, \cdot)$ is an inverse semigroup. To verify that $S \times T$ is a left inverse semi-brace, i.e., (1) holds, the computations are analogue to those in the proof of [16, Theorem 6], by using the fact that

$$
(a, u)^{-1}=\left(\alpha_{\beta_{a}^{-1}(u)}^{-1}\left(a^{-1}\right), \beta_{\alpha_{u}^{-1}(a)}^{-1}\left(u^{-1}\right)\right)
$$

Therefore, the claim follows.

Remark 5. We observe that the map $\varphi: S \times T \rightarrow S \times T,(a, u) \mapsto\left(a, \beta_{a}(u)\right)$ is an isomorphism of inverse semigroups from the Zappa product of $S$ and $T$ into the semigroup $(S \times T, \cdot)$. Indeed, clearly, $\varphi$ is an isomorphism of semigroups and

$$
\begin{aligned}
\varphi\left((a, u)^{-1}\right) & =\varphi\left(\alpha_{\beta_{a}^{-1}(u)}^{-1}\left(a^{-1}\right), \beta_{\alpha_{u}^{-1}(a)}^{-1}\left(u^{-1}\right)\right) \\
& =\left(\alpha_{\beta_{a}^{-1}(u)}^{-1}\left(a^{-1}\right), \beta_{\alpha_{\beta_{a}^{-1}(u)}^{-1}\left(a^{-1}\right)}^{-1} \beta_{\alpha_{u}^{-1}(a)}^{-1}\left(u^{-1}\right)\right) \\
& =\left(a, \beta_{a}^{-1}(u)\right)^{-1} \\
& =(\varphi(a, u))^{-1},
\end{aligned}
$$


for every $(a, u) \in S \times T$.

Similarly to [16, Remark 5], by (4), one can check the following equalities.

Lemma 13. Let $(S, T, \alpha, \beta)$ be a matched product system of left inverse semi-braces. Then, they hold

$$
\begin{aligned}
& \lambda_{a} \alpha_{\beta_{a}^{-1}(u)}=\alpha_{u} \lambda_{\alpha_{u}^{-1}(a)} \\
& \lambda_{u} \beta_{\alpha_{u}^{-1}(a)}=\beta_{a} \lambda_{\beta_{a}^{-1}(u)}
\end{aligned}
$$

for all $a \in S$ and $u \in T$.

Now, to show that the map $r_{S \bowtie T}$ associated to a matched product $S \bowtie T$ is a solution, let us recall the notion of matched product system of solutions introduced in [15]. Given a solution $r_{S}$ on a set $S$ and a solution $r_{T}$ on a set $T$, if $\alpha: T \rightarrow \operatorname{Sym}(S)$ and $\beta: S \rightarrow \operatorname{Sym}(T)$ are maps, set $\alpha_{u}:=\alpha(u)$, for every $u \in T$, and $\beta_{a}:=\beta(a)$, for every $a \in S$, then the quadruple $\left(r_{S}, r_{T}, \alpha, \beta\right)$ is said to be a matched product system of solutions if the following conditions hold

$$
\begin{gathered}
\alpha_{u} \alpha_{v}=\alpha_{\lambda_{u}(v)} \alpha_{\rho_{v}(u)} \\
\rho_{\alpha_{u}^{-1}(b)} \alpha_{\beta_{a}(u)}^{-1}(a)=\alpha_{\beta_{\rho_{b}(a)}^{-1}}^{\beta_{b}^{-1}(u)} \rho_{b}(a) \\
\lambda_{a} \alpha_{\beta_{a}^{-1}(u)}=\alpha_{u} \lambda_{\alpha_{u}^{-1}(a)}
\end{gathered}
$$

$$
\begin{gathered}
\beta_{a} \beta_{b}=\beta_{\lambda_{a}(b)} \beta_{\rho_{b}(a)} \\
\rho_{\beta_{a}^{-1}(v)} \beta_{\alpha_{u}(a)}^{-1}(u)=\beta_{\alpha_{\rho_{v}(u)^{-1}} \alpha_{v}^{-1}(a)} \rho_{v}(u) \\
\lambda_{u} \beta_{\alpha_{u}^{-1}(a)}=\beta_{a} \lambda_{\beta_{a}^{-1}(u)}
\end{gathered}
$$

for all $a, b \in S$ and $u, v \in T$.

As shown in [15, Theorem 2], any matched product system of solutions determines a new solution on the set $S \times T$. Theorem 14 (Theorem 2, [15]). Let $\left(r_{S}, r_{T}, \alpha, \beta\right)$ be a matched product system of solutions. Then, the map $r: S \times T \times S \times T \rightarrow S \times T \times S \times T$ defined by

$$
r((a, u),(b, v)):=\left(\left(\alpha_{u} \lambda_{\bar{a}}(b), \beta_{a} \lambda_{\bar{u}}(v)\right),\left(\alpha_{\bar{U}}^{-1} \rho_{\alpha_{\bar{u}}(b)}(a), \beta_{\bar{A}}^{-1} \rho_{\beta_{\bar{a}}(v)}(u)\right)\right)
$$

where we set

$$
\bar{a}:=\alpha_{u}^{-1}(a), \quad \bar{u}:=\beta_{a}^{-1}(u), \quad A:=\alpha_{u} \lambda_{\bar{a}}(b), \quad U:=\beta_{a} \lambda_{\bar{u}}(v), \bar{A}:=\alpha_{U}^{-1}(A), \bar{U}:=\beta_{A}^{-1}(U),
$$

for all $(a, u),(b, v) \in S \times T$, is a solution. This solution is called the matched product of the solutions $r_{S}$ and $r_{T}$ (via $\alpha$ and $\beta)$ and it is denoted by $r_{S} \bowtie r_{T}$.

If $\left(r_{S}, r_{T}, \alpha, \beta\right)$ is a matched product system of solutions, we denote $\alpha_{u}^{-1}(a)$ with $\bar{a}$ and $\beta_{a}^{-1}(u)$ with $\bar{u}$, when the pair $(a, u) \in S \times T$ is clear from the context.

Theorem 15. Let $(S, T, \alpha, \beta)$ be a matched product system of left inverse semi-braces, $r_{S}$ and $r_{T}$ solutions on $S$ and $T$, respectively. Then, $r_{S \bowtie T}$ is a solution on the matched product $S \bowtie T$ and $r_{S \bowtie T}=r_{S} \bowtie r_{T}$. 
Proof. Initially, we compute the components of $r_{S \bowtie T}$ and we show that they are exactly those of the matched solution of $r_{S}$ and $r_{T}$ as in 111 . Specifically, if $(a, u),(b, v) \in S \times T$, we prove that

$$
\lambda_{(a, u)}(b, v)=\left(\alpha_{u} \lambda_{\bar{a}}(b), \beta_{a} \lambda_{\bar{u}}(v)\right) \quad \rho_{(b, v)}(a, u)=\left(\alpha_{\bar{U}}^{-1} \rho_{\alpha_{\bar{u}}(b)}(a), \beta_{\bar{A}}^{-1} \rho_{\beta_{\bar{a}}(v)}(u)\right) .
$$

Firstly,

$$
\begin{aligned}
\lambda_{(a, u)}(b, v) & =(a, u)\left(\left(\alpha_{\bar{u}}^{-1}\left(a^{-1}\right), \beta_{\bar{a}}^{-1}\left(u^{-1}\right)\right)+(b, v)\right) \\
& =(a, u)\left(\left(\alpha_{\bar{u}}^{-1}\left(a^{-1}\right)+b, \beta_{\bar{a}}^{-1}\left(u^{-1}\right)+v\right)\right) \\
& =\left(a \alpha_{\bar{u}}\left(\alpha_{\bar{u}}^{-1}\left(a^{-1}\right)+b\right), u \beta_{\bar{a}}\left(\beta_{\bar{a}}^{-1}\left(u^{-1}\right)+v\right)\right) \\
& =\left(a\left(a^{-1}+\alpha_{\bar{u}}(b)\right), u\left(u^{-1}+\beta_{\bar{a}}(v)\right)\right) \\
& =\left(\lambda_{a} \alpha_{\bar{u}}(b), \lambda_{u} \beta_{\bar{a}}(v)\right) \\
& =\left(\alpha_{u} \lambda_{\bar{a}}(b), \beta_{a} \lambda_{\bar{u}}(v)\right)
\end{aligned}
$$

Moreover, set

$$
x:=\alpha_{\bar{u}}^{-1}\left(a^{-1}+\alpha_{\bar{u}}(b)\right), \quad y:=\beta_{\bar{a}}^{-1}\left(u^{-1}+\beta_{\bar{a}}(v)\right), \quad \mathcal{A}:=\alpha_{y}^{-1}(x), \quad \mathcal{U}:=\beta_{x}^{-1}(y),
$$

we have that

$$
\begin{array}{rlr}
\rho_{(b, v)}(a, u) & =\left(\left(\alpha_{\bar{u}}^{-1}\left(a^{-1}\right), \beta_{\bar{a}}^{-1}\left(u^{-1}\right)\right)+(b, v)\right)^{-1}(b, v) & \text { by (ㅁ) } \\
& =\left(\alpha_{\bar{u}}^{-1}\left(a^{-1}\right)+b, \beta_{\bar{a}}^{-1}\left(u^{-1}\right)+v\right)^{-1}(b, v) & \\
& =(x, y)^{-1}(b, v) & \text { by }(8) \\
& =\left(\alpha_{\beta_{x}^{-1}(y)}^{-1}\left(x^{-1}\right), \beta_{\alpha_{y}^{-1}(x)}^{-1}\left(y^{-1}\right)\right)(b, v) & \text { by (6)-(7) } \\
& =\left(\mathcal{A}^{-1}, \mathcal{U}^{-1}\right)(b, v) & \\
& =\left(\mathcal{A}^{-1} \alpha_{\beta_{\mathcal{A}^{-1}}^{-1}\left(\mathcal{U}^{-1}\right)}(b), \mathcal{U}^{-1} \beta_{\alpha_{\mathcal{U}^{-1}}^{-1}\left(\mathcal{A}^{-1}\right)}(v)\right) . &
\end{array}
$$

Now, we focus on the first component and we show that it is equal to $\alpha_{\bar{U}}^{-1} \rho_{\alpha_{\bar{u}}(b)}(a)$. By $(7)$, we get

$$
\mathcal{A}=\alpha_{\bar{u}^{-1}+v}^{-1}(x)=\alpha_{\bar{u}\left(\bar{u}^{-1}+v\right)}^{-1}\left(a^{-1}+\alpha_{\bar{u}}(b)\right)=\alpha_{\lambda_{\bar{u}}(v)}^{-1}\left(a^{-1}+\alpha_{\bar{u}}(b)\right)
$$

and, similarly, by (6),

$$
\mathcal{U}=\beta_{\lambda_{\bar{a}}(b)}^{-1}\left(u^{-1}+\beta_{\bar{a}}(v)\right)
$$


Hence,

$$
\begin{array}{rlr}
\mathcal{A}^{-1} & =\alpha_{\beta_{a^{-1}+\alpha_{\bar{u}}(b)}^{-1} \lambda_{\bar{u}}(v)}^{-1}\left(\left(a^{-1}+\alpha_{\bar{u}}(b)\right)^{-1}\right) & \text { by Remark } 6 \\
& =\alpha_{\beta_{a^{-1} a_{a}\left(a^{-1}+\alpha_{\bar{u}}(b)\right.}^{-1}}^{-1} \lambda_{\bar{u}(v)}\left(\left(a^{-1}+\alpha_{\bar{u}}(b)\right)^{-1}\right) \\
& =\alpha_{\beta_{\lambda_{a} \alpha_{\bar{u}}(b)}^{-1} \beta_{a} \lambda_{\bar{u}}(v)}^{-1}\left(\left(a^{-1}+\alpha_{\bar{u}}(b)\right)^{-1}\right) \\
& =\alpha_{\beta_{\alpha_{u} \lambda_{\bar{a}}(b)}^{-1} \beta_{a} \lambda_{\bar{u}}(v)}^{-1}\left(\left(a^{-1}+\alpha_{\bar{u}}(b)\right)^{-1}\right) \\
& =\alpha_{\bar{U}}^{-1}\left(\left(a^{-1}+\alpha_{\bar{u}}(b)\right)^{-1}\right) .
\end{array}
$$

By (4), it follows that

$$
\mathcal{A}^{-1} \alpha_{\beta_{\mathcal{A}^{-1}}^{-1}\left(\mathcal{U}^{-1}\right)}(b)=\alpha_{\bar{U}}^{-1}\left(\left(a^{-1}+\alpha_{\bar{u}}(b)\right)^{-1} \alpha_{\left.\beta_{\left(a^{-1}+\alpha \bar{u}\right.}^{-1}(b)\right)^{-1}}(\bar{U})^{\left.\alpha_{\beta_{\mathcal{A}^{-1}}^{-1}\left(\mathcal{U}^{-1}\right)}(b)\right)}\right)
$$

and so, set $\mathcal{B}:=\alpha_{\beta_{\left(a^{-1}+\alpha_{\bar{u}}(b)\right)^{-1}}^{-1}(\bar{U})} \alpha_{\beta_{\mathcal{A}^{-1}}^{-1}\left(\mathcal{U}^{-1}\right)}(b)$ the thesis reduces to show that

$$
\mathcal{B}=\alpha_{\bar{u}}(b)
$$

We obtain that the subscript of the first $\alpha$ in $\mathcal{B}$ becomes

$$
\begin{array}{rlr}
\beta_{\left(a^{-1}+\alpha_{\bar{u}}(b)\right)^{-1}}^{-1}(\bar{U}) & =\beta_{a^{-1}+\alpha_{\bar{u}}(b)}(\bar{U}) & \\
& =\beta_{a^{-1} a\left(a^{-1}+\alpha_{\bar{u}}(b)\right)}(\bar{U}) & \text { by Remark 4 } \\
& =\beta_{a}^{-1} \beta_{\lambda_{a} \alpha_{\bar{u}(b)}} \beta_{\lambda_{a} \alpha_{\bar{u}(b)}}^{-1} \lambda_{u} \beta_{\bar{a}}(v) & \\
& =\beta_{a}^{-1} \lambda_{u} \beta_{\bar{a}}(v) & \text { by (10) } \\
& =\lambda_{\bar{u}}(v) &
\end{array}
$$

and so

$$
\mathcal{B}=\alpha_{\lambda_{\bar{u}}(v)} \alpha_{\beta_{\mathcal{A}^{-1}}^{-1}\left(\mathcal{U}^{-1}\right)}(b)=\alpha_{\bar{u}} \alpha_{\bar{u}^{-1}+v} \alpha_{\left(\beta_{\mathcal{A}^{-1}}^{-1}\left(\mathcal{U}^{-1}\right)\right)^{-1}}^{-1}(b) .
$$

Thus, we show that $\left(\beta_{\mathcal{A}^{-1}}^{-1}\left(\mathcal{U}^{-1}\right)\right)^{-1}=\bar{u}^{-1}+v$. To this aim, note that, by Remark 4 , it holds

$$
\beta_{\lambda_{\bar{a}}(b)}^{-1}\left(u^{-1}+\beta_{\bar{a}}(v)\right)=\beta_{\lambda_{\bar{a}}(b)}^{-1} \beta_{\bar{a}}\left(\beta_{\bar{a}}^{-1}\left(u^{-1}\right)+v\right)=\beta_{\bar{a}^{-1}+b}^{-1}\left(\bar{u}^{-1}+v\right)
$$

and, analogously,

$$
\alpha_{\lambda_{\bar{u}}(v)}^{-1}\left(a^{-1}+\alpha_{\bar{u}}(b)\right)=\alpha_{\bar{u}^{-1}+v}^{-1}\left(\bar{a}^{-1}+b\right) .
$$


We compute

$$
\begin{aligned}
& \left(\beta_{\mathcal{A}^{-1}}^{-1}\left(\mathcal{U}^{-1}\right)\right)^{-1} \\
& =\beta_{\left(\alpha_{\lambda_{\bar{u}}(v)}^{-1}\left(a^{-1}+\alpha_{\bar{u}}(b)\right)\right)^{-1}}^{-1}\left(\beta_{\lambda_{\bar{a}}(b)}^{-1}\left(u^{-1}+\beta_{\bar{a}}(v)\right)\right)^{-1} \\
& =\beta_{\alpha_{\left(\beta_{\lambda_{\bar{a}}(b)}^{-1}\right.}^{-1}}^{\left.\left.\alpha^{-1}+\beta_{\bar{a}}(v)\right)\right)^{-1}}\left(\alpha_{\lambda_{\bar{u}}(v)}^{-1}\left(a^{-1}+\alpha_{\bar{u}}(b)\right)\right)^{-1} \beta_{\lambda_{\bar{a}}(b)}^{-1}\left(u^{-1}+\beta_{\bar{a}}(v)\right) \\
& =\beta_{\alpha_{\left(\beta_{\bar{a}-1}-1\right.}^{-1}}^{\alpha^{-1}} \\
& =\beta_{\left.\left.\left(\bar{a}^{-1}+b\right)^{-1}+v\right)\right)^{-1}}^{-1}\left(\alpha_{\bar{a}^{-1}+v^{-1}+b}^{-1}\left(\bar{u}^{-1}+v\right)\right. \\
& =\bar{u}^{-1}+v,
\end{aligned}
$$

which proves what stated before. By reversing the role of $\alpha$ and $\beta$, we obtain with similar computations that the second component of $\rho_{(b, v)}(a, u)$ is equal to $\beta_{\bar{A}}^{-1} \rho_{\beta_{\bar{a}}(v)}(u)$.

Now, to get the claim, it remains to be proven that $\left(r_{S}, r_{T}, \alpha, \beta\right)$ is matched product system of solutions, by verifying only (s1), (s3), and (s5), since the the other ones can be obtained similarly. Initially, note that, by Remark 4

$$
\alpha_{\lambda_{u}(v)} \alpha_{\rho_{v}(u)}=\alpha_{\lambda_{u}(v) \rho_{v}(u)}=\alpha_{u\left(u^{-1}+v\right)\left(u^{-1}+v\right)^{-1} v}=\alpha_{u} \alpha_{\left(u^{-1}+v\right)\left(u^{-1}+v\right)^{-1}} \alpha_{v}=\alpha_{u} \alpha_{v}=\alpha_{u v},
$$

hence (s1) holds. Moreover,

$$
\begin{aligned}
\rho_{\alpha_{u}^{-1}(b)} \alpha_{\beta_{a}(u)}^{-1}(a) & =\rho_{\alpha_{u}^{-1}(b)}\left(\left(\alpha_{u}^{-1}\left(a^{-1}\right)\right)^{-1}\right) \\
& =\left(\alpha_{u}^{-1}\left(a^{-1}\right)+\alpha_{u}^{-1}(b)\right)^{-1} \alpha_{u}^{-1}(b) \\
& =\left(\alpha_{u}^{-1}\left(a^{-1}+b\right)\right)^{-1} \alpha_{u}^{-1}(b) \\
& =\left(\left(\alpha_{u}^{-1}(b)\right)^{-1} \alpha_{u}^{-1}\left(a^{-1}+b\right)\right)^{-1} \\
& =\left(\alpha_{\beta_{b}^{-1}(u)}^{-1}\left(b^{-1}\right) \alpha_{u}^{-1}\left(a^{-1}+b\right)\right)^{-1} \\
& =\left(\alpha_{\beta_{b}^{-1}(u)}^{-1}\left(b^{-1} \alpha_{\beta_{b-1}^{-1} \beta_{b}^{-1}(u)}^{-1} \alpha_{u}^{-1}\left(a^{-1}+b\right)\right)\right)^{-1} \\
& =\left(\alpha_{\beta_{b}^{-1}(u)}^{-1}\left(b^{-1}\left(a^{-1}+b\right)\right)\right)^{-1} \\
& =\left(\alpha_{\beta_{b}^{-1}(u)}^{-1}\left(\rho_{b}(a)\right)^{-1}\right)^{-1} \\
& =\alpha_{\beta_{\rho_{b}(a)}^{-1} \beta_{b}^{-1}(u)} \rho_{b}(a)
\end{aligned}
$$

hence (53) is satisfied. Finally, it is clear that (55) coincides with (9).

Therefore, by Theorem 14, the claim follows. 
In the next, we focus on a particular case of the previous construction, the semidirect product of two inverse semibraces. From now on, given a matched product system $(S, T, \alpha, \beta)$, we consider $\beta_{a}=\mathrm{id}_{T}$, for every $a \in S$. In this way, the inverse semigroup $(S \times T, \cdot)$ is exactly the semidirect product of the inverse semigroups $(S, \cdot)$ and $(T, \cdot)$ via $\alpha$, in the sense of [42] and [45]. Let us note that this semidirect product is a particular case of the Zappa product of two semigroups with ${ }^{u} a=\sigma(u)(a)=\alpha_{u}(a)$ and $u^{a}=\beta_{a}(u)=u$, for all $a \in S$ and $u \in T$. Specifically, the multiplication $\cdot$ on $S \times T$ is given by

$$
(a, u)(b, v)=\left(a^{u} b, u v\right)
$$

for all $(a, u),(b, v) \in S \times T$.

Let us recall the result essentially contained in [45, Theorem 6] that, in contrast to Theorem 9, is a characterization to obtain an inverse semigroup.

Theorem 16. Let $S, T$ be semigroups and $\sigma: T \rightarrow \operatorname{End}(S)$ a homomorphism. Then, the semidirect product of $S$ and $T$ via $\sigma$ is an inverse semigroup if and only if they hold

1. S and T are inverse semigroups;

2. $\sigma(T) \subseteq \operatorname{Aut}(S)$.

Let us note that condition 2. in the previous theorem is equivalent to the property

$$
{ }^{e} a=a,
$$

for all $e \in E(T)$ and $a \in S$. Such a condition derives also from Remark 4

As a consequence of (16), $\sigma$ is a homomorphism of inverse semigroups, namely, in addition, it holds $\sigma\left(u^{-1}\right)=$ $\sigma(u)^{-1}$, for every $u \in T$. Moreover, one can check that

$$
(a, u)^{-1}=\left(u^{-1} a^{-1}, u^{-1}\right)
$$

for every $(a, u) \in S \times T$, which follows also from Lemma 11

The following is an example of semidirect product of two Clifford semigroups which is not a Clifford semigroup. Specifically, this shows that left inverse semi-braces can be obtained also starting from two particular generalized left semi-braces, as one can concretely see later in Example 14

Example 9. Considered the set $X:=\{1, x, y\}$, let $S$ be the upper semilattice on $X$ with join 1 and $T$ the commutative inverse monoid with identity 1 for which $x x=y y=x$ and $x y=y$. If $\tau$ is the automorphism of $S$ given by the transposition $\tau:=(x y)$, then the map $\sigma: T \rightarrow \operatorname{Aut}(S)$ given by $\sigma(1)=\sigma(x)=\operatorname{id}_{S}$ and $\sigma(y)=\tau$, is a homomorphism from $T$ into $\operatorname{Aut}(S)$. Therefore, by Theorem 16 , it follows that the semidirect product of $S$ and $T$ via $\sigma$ is an inverse semigroup. Observe that such a semigroup is not a Clifford semigroup. Indeed, since by (17) it holds that $(x, y)^{-1}=(y, y)$, we have $(x, y)(x, y)^{-1}=(x, x)$, but $(x, y)^{-1}(x, y)=(y, x)$. 
The following result is a consequence of Theorem 12

Corollary 17. Let $S, T$ be left inverse semi-braces and $\sigma: T \rightarrow \operatorname{Aut}(S)$ a homomorphism from $(T, \cdot)$ into the automorphism group of the left inverse semi-brace $S$. Then, the structure $(S \times T,+, \cdot)$ where

$$
\begin{aligned}
(a, u)+(b, v) & :=(a+b, u+v) \\
(a, u)(b, v) & :=\left(a^{u} b, u v\right),
\end{aligned}
$$

for all $(a, u),(b, v) \in S \times T$, is a left inverse semi-brace. We call such an inverse semi-brace the semidirect product of $S$ and $T$ via $\sigma$ and we denote it by $S \rtimes_{\sigma} T$.

Example 10. Let $X:=\{1, x, y\}$ be a set, $S$ the cyclic group on $X$, and $T$ the commutative inverse monoid on $X$ in Example 9 Considered $\iota: S \rightarrow S$ the automorphism of $S$ defined by $\iota(a)=-a$, for every $a \in S$, we obtain that the map $\sigma: T \rightarrow \operatorname{Aut}(S)$ given by $\sigma(1)=\sigma(x)=\operatorname{id}_{S}$, and $\sigma(y)=\iota$ is a homomorphism from $T$ into $\operatorname{Aut}(S)$. Therefore, by Theorem 16, it follows that the semidirect product of $S$ and $T$ via $\sigma$ is an inverse semigroup (which is a Clifford semigroup). Now, set $a+b=a$, for all $a, b \in S$, and $u+v=u v$, for all $u, v \in T$, then $S$ is a left semi-brace and, as seen in 1. of Examples 4, $T$ is a generalized left semi-brace. In addition, the map $\sigma$ is a homomorphism from $T$ into the automorphism of the inverse semi-brace $S$. Therefore, by Corollary 17, $S \times T$ endowed with the following operations

$$
(a, u)+(b, v)=(a, u v) \quad(a, u)(b, v)=\left(a^{u} b, u v\right)
$$

for all $(a, u),(b, v) \in S \times T$, is a left inverse semi-brace that is the semidirect product of $S$ and $T$ via $\sigma$. In particular, $S \rtimes_{\sigma} T$ is a generalized left semi-brace.

The semidirect product of two generalized semi-braces can be an inverse semi-brace which is not a generalized semi-brace.

Example 11. Considered the inverse semigroups $(S, \cdot)$ and $(T, \cdot)$ in the Example 9 set $a+b=b$, for all $a, b \in S$, and $u+v=u v$, for all $u, v \in T$, then $S$ is a left semi-brace and $T$ is a generalized semi-brace. Furthermore, the map $\sigma: T \rightarrow \operatorname{Aut}(S)$ defined by $\sigma(1)=\sigma(x)=\operatorname{id}_{S}$ and $\sigma(y)=\tau$, is a homomorphism from $T$ into the automorphism of the inverse semi-brace $S$. Therefore, by Corollary $17, S \times T$ endowed with the following operations

$$
(a, u)+(b, v)=(b, u v) \quad(a, u)(b, v)=\left(a^{u} b, u v\right)
$$

for all $(a, u),(b, v) \in S \times T$, is a left inverse semi-brace that is the semidirect product of $S$ and $T$ via $\sigma$.

As a consequence of Theorem 15, given two solutions $r_{S}$ and $r_{T}$ on two left inverse semi-braces $S$ and $T$, respectively, the map $r_{B}$ associated to a semidirect product $B:=S \rtimes_{\sigma} T$ via a homomorphism $\sigma: T \rightarrow \operatorname{Aut}(S)$ is still a solution. 
Corollary 18. Let $S, T$ be left inverse semi-braces, $r_{S}$ and $r_{T}$ solutions on $S$ and $T$, respectively, and $\sigma: T \rightarrow \operatorname{Aut}(S)$ a homomorphism from $T$ into the automorphism group of the inverse semi-brace $S$. Then, the map $r_{B}$ associated to the semidirect product $B:=S \rtimes_{\sigma} T$, given by

$$
r_{B}((a, u),(b, v))=\left(\left({ }^{u} \lambda_{u^{-1} a}(b), \lambda_{u}(v)\right),\left(\lambda_{u}(v)^{-1} \rho_{u_{b}}(a), \rho_{v}(u)\right)\right)
$$

for all $(a, u),(b, v) \in S \times T$, is a solution. In particular, such a solution $r_{B}$ is actually the semidirect product of the solutions $r_{S}$ and $r_{T}$.

Example 12. Let $S, T$ be the trivial left inverse semi-braces in Example 1 with $(S,+)$ a left zero semigroup and $(T,+)$ a right zero semigroup, and $\sigma: T \rightarrow \operatorname{Aut}(S)$ a homomorphism from $(T, \cdot)$ into the automorphism group of the left inverse semi-brace $S$. Then, by Corollary $17,=S \rtimes_{\sigma} T$ is a left inverse semi-brace and, by Corollary 18 the map $r_{B}$ associated to $B$ given by

$$
r_{B}((a, u),(b, v))=\left(\left(a a^{-1}, u v\right),\left(v^{-1}\left(a^{u} b\right), v^{-1} v\right)\right)
$$

is a solution. Specifically, it is exactly the semidirect product of the solution $r_{S}$ and $r_{T}$ in Examples 5 given by $r_{S}(a, b)=\left(a a^{-1}, a b\right)$ and $r_{T}(u, v)=\left(u v, v^{-1} v\right)$, respectively. Moreover, $r_{B}$ is idempotent, consistently with [15, Corollary 5].

To analyze the next examples, let us recall the notions of index and period of a solution $r$ introduced in [16] that are respectively defined as

$$
\begin{aligned}
& \mathrm{i}(r):=\min \left\{j \mid j \in \mathbb{N}_{0}, \exists l \in \mathbb{N} r^{j}=r^{l}, j \neq l\right\} \\
& \mathrm{p}(r):=\min \left\{k \mid k \in \mathbb{N}, r^{\mathrm{i}(r)+k}=r^{\mathrm{i}(r)}\right\} .
\end{aligned}
$$

These definitions of the index and the order are slightly different from the classical ones (cf. [33, p. 10]). This choice is functional to distinguish bijective solutions, having index 0 , from non-bijective ones, having index a positive integer.

Example 13. Let $B:=S \rtimes_{\sigma} T$ be the left inverse semi-brace in Example 10, Then, by (16), the map $r_{B}$ associated to $B$ is given by

$$
r_{B}((a, u),(b, v))=\left(\left(1, u u^{-1} v\right),\left(v^{-1}\left(a^{u} b\right), u v^{-1} v\right)\right)
$$

and, by Corollary 18 it is a solution. Specifically, $r_{B}$ is the semidirect product of the solutions $r_{S}(a, b)=(1, a b)$ on $S$ (cf. Examples 5) and $r_{T}(u, v)=\left(u u^{-1} v, u v^{-1} v\right)$ on $T$ (see Examples 8), respectively. Moreover, by [16, Proposition 10], such a solution $r_{B}$ is cubic with $\mathrm{i}\left(r_{B}\right)=1$ and $\mathrm{p}\left(r_{B}\right)=2$. 
Example 14. Let $B:=S \rtimes_{\sigma} T$ be the left inverse semi-brace in Example 11. Then, by 16, the map $r_{B}$ associated to $B$ is given by

$$
r_{B}((a, u),(b, v))=\left(\left(a^{u} b, u u^{-1} v\right),\left(v^{-1} u\left(b^{-1} b\right), u v^{-1} v\right)\right)
$$

and, by Corollary 18, it is a solution. In particular, $r_{B}$ is the semidirect product of the solutions $r_{S}(a, b)=\left(a b, b^{-1} b\right)$ on $S$ (cf. Examples 5) and $r_{T}(u, v)=\left(u u^{-1} v, u v^{-1} v\right)$ on $T$ (see Examples 8), respectively. Furthermore, by [16, Proposition 10], such a solution $r_{B}$ is cubic with $\mathrm{i}\left(r_{B}\right)=1$ and $\mathrm{p}\left(r_{B}\right)=2$.

\section{The double semidirect product of left inverse semi-braces}

This section is devoted to present a new construction of left inverse semi-braces which includes the semidirect product in Corollary 17, that is the double semidirect product. In particular, we show that under mild assumptions the map associated to the double semidirect product of arbitrary left semi-braces is a solution.

Theorem 19. Let $S$ and $T$ be two left inverse semi-braces, $\sigma: T \rightarrow \operatorname{Aut}(S)$ a homomorphism from $(T, \cdot)$ into the automorphism group of the left inverse semi-brace $S$, with ${ }^{u} a:=\sigma(u)(a)$, for all $a \in S$ and $u \in T$, and $\delta: S \rightarrow$ End $(T)$ an anti-homomorphism from $(S,+)$ into the endomorphism semigroup of $(T,+)$, with $u^{a}:=\delta(a)(u)$, for all $a \in S$ and $u \in T$. If the condition

$$
(u v)^{\lambda_{a}\left({ }^{u} b\right)}+u\left(\left(u^{-1}\right)^{b}+w\right)=u\left(v^{b}+w\right)
$$

holds, for all $a, b \in S$ and $u, v, w \in T$, then $S \times T$ with respect to

$$
\begin{aligned}
(a, u)+(b, v) & :=\left(a+b, u^{b}+v\right) \\
(a, u)(b, v) & :=\left(a^{u} b, u v\right)
\end{aligned}
$$

for all $(a, u),(b, v) \in S \times T$, is a left inverse semi-brace. We call such a left inverse semi-brace the double semidirect product of $S$ and $T$ via $\sigma$ and $\delta$.

Proof. At first, note that the structure $(S \times T,+)$ is a semigroup since it is exactly the semidirect product of the semigroup $(S,+)$ and $(T,+)$ via $\delta$. Moreover, by Theorem $16,(S \times T, \cdot)$ is an inverse semigroup. Thus, it only remains to prove that (1) is satisfied. If $(a, u),(b, v),(c, w) \in S \times T$, we obtain

$$
(a, u)((b, v)+(c, w))=(a, u)\left(b+c, v^{c}+w\right)=\left(a^{u}(b+c), u\left(v^{c}+w\right)\right)
$$


and

$$
\begin{aligned}
(a, u)(b, v)+ & (a, u)\left((a, u)^{-1}+(c, w)\right) \\
& =\left(a^{u} b, u v\right)+(a, u)\left(\left(u^{-1} a^{-1}, u^{-1}\right)+(c, w)\right) \\
& =\left(a^{u} b, u v\right)+(a, u)\left(u^{-1} a^{-1}+c,\left(u^{-1}\right)^{c}+w\right) \\
& =\left(a^{u} b, u v\right)+\left(a^{u}\left(u^{-1} a^{-1}+c\right), u\left(\left(u^{-1}\right)^{c}+w\right)\right) \\
& =\left(a^{u} b, u v\right)+\left(a\left(a^{-1}+{ }^{u} c\right), u\left(\left(u^{-1}\right)^{c}+w\right)\right) \\
& =\left(a^{u} b+\lambda_{a}\left({ }^{u} c\right),(u v)^{\lambda_{a}\left({ }^{u} c\right)}+u\left(\left(u^{-1}\right)^{c}+w\right)\right) \\
& =\left(a\left({ }^{u} b+{ }^{u} c\right),(u v)^{\lambda_{a}\left({ }^{u} c\right)}+u\left(\left(u^{-1}\right)^{c}+w\right)\right),
\end{aligned}
$$

hence, by 18 the claim follows.

We specialize the Theorem 19 for (left cancellative) left semi-braces and skew left braces.

Corollary 20. Let $S, T$ be (left cancellative) left semi-braces, $\sigma: T \rightarrow \operatorname{Aut~}(S)$ a homomorphism from $(T, \cdot)$ into the automorphism group of $(S,+, \cdot)$, and $\delta: S \rightarrow$ End $(T)$ an anti-homomorphism from $(S,+)$ into the automorphism group of $(T,+)$ satisfying (18). Then, the double semidirect product of $S$ and $T$ via $\sigma$ and $\delta$ is a (left cancellative) semi-bracee.

We remind that a skew left brace is a triple $(S,+, \cdot)$ where $(S,+)$ and $(S, \cdot)$ are groups, see [31]. Let us note that given two skew left braces $S$ and $T$, the double semidirect product of $S$ and $T$ is not necessarily a skew left braces, too. Indeed, it is a consequence of the fact that the semidirect product of two groups $(S,+)$ and $(T,+)$ with $\delta(T) \nsubseteq \operatorname{Aut}(T)$ in general is not a group, see [45].

Corollary 21. Let $S, T$ be skew left braces, $\sigma: T \rightarrow \operatorname{Aut}(S)$ a homomorphism from $(T, \cdot)$ into the automorphism group of $(S,+, \cdot)$, and $\delta: S \rightarrow$ Aut $(T)$ an anti-homomorphism from $(S,+)$ into the automorphism group of $(T,+)$ satisfying (18). Then, the double semidirect product of $S$ and $T$ via $\sigma$ and $\delta$ is a skew left brace.

Now, our aim is to study the solution associated to any double semidirect product. For convenience, to calculate such a map, in the following lemma we rewrite conditions (9)-(10) in the exponential notation.

Lemma 22. Let $S, T$ be a left inverse semi-brace and $\sigma: T \rightarrow \operatorname{Aut}(S)$ a homomorphism from $(T, \cdot)$ into the automorphism of the inverse semi-brace S. Then, the following properties are satisfied:

$$
\begin{aligned}
& \text { 1. }{ }^{u} \lambda_{a}(b)=\lambda_{u_{a}}\left({ }^{u} b\right) \\
& \text { 2. }{ }^{u} \rho_{b}(a)=\rho^{u_{b}}\left({ }^{u} a\right) \text {, }
\end{aligned}
$$

for all $a, b \in S$ and $u \in T$. 
To simplify our computations concerning the map associated to a double semidirect product, hereinfater we use the notation

$$
\Omega_{u, v}^{a}:=\left(u^{-1}\right)^{a}+v
$$

for all $a \in S, u, v \in T$.

Proposition 23. Let $S, T$ be left inverse semi-braces and $B$ the double semidirect product of $S$ and $T$ via $\sigma$ and $\delta$. Then, the map $r_{B}$ associated to $B$ is given by

$$
r_{B}((a, u),(b, v))=\left(\left(\lambda_{a}\left({ }^{u} b\right), u \Omega_{u, v}^{b}\right),\left(\left(\Omega_{u, v}^{b}\right)^{-1} u^{-1} \rho_{u b}(a),\left(\Omega_{u, v}^{b}\right)^{-1} v\right)\right)
$$

for all $(a, u),(b, v) \in S \times T$.

Proof. Let us compute the components of the map $r_{B}$. If $a, b \in S$ and $u, v \in T$, by 17, we have that

$$
\lambda_{(a, u)}(b, v)=\left(a^{u}\left(u^{u^{-1}} a^{-1}+b\right), u \Omega_{u, v}^{b}\right)=\left(a\left(a^{-1}+{ }^{u} b\right), u \Omega_{u, v}^{b}\right)=\left(\lambda_{a}\left({ }^{u} b\right), u \Omega_{u, v}^{b}\right)
$$

and, by 17 and Lemma 22

$$
\begin{aligned}
\rho_{(b, v)}(a, u) & =\left(\left(\Omega_{u, v}^{b}\right)^{-1}\left(u^{-1} a^{-1}+b\right)^{-1}\left(\Omega_{u, v}^{b}\right)^{-1} b,\left(\Omega_{u, v}^{b}\right)^{-1} v\right) \\
& =\left(\left(\Omega_{u, v}^{b}\right)^{-1}\left(\left(u^{-1} a^{-1}+b\right)^{-1} b\right),\left(\Omega_{u, v}^{b}\right)^{-1} v\right) \\
& =\left(\left(\Omega_{u, v}^{b}\right)^{-1} \rho_{b}\left(\left(u^{-1} a^{-1}\right)^{-1}\right),\left(\Omega_{u, v}^{b}\right)^{-1} v\right) \\
& =\left(\left(\Omega_{u, v}^{b}\right)^{-1} \rho_{b}\left(u^{-1} a\right),\left(\Omega_{u, v}^{b}\right)^{-1} v\right) \\
& =\left(\left(\Omega_{u, v}^{b}\right)^{-1} u^{-1} \rho_{u b}(a),\left(\Omega_{u, v}^{b}\right)^{-1} v\right) .
\end{aligned}
$$

Therefore, the claim follows.

Note that the second component of $\lambda_{(a, u)}(b, v)$ and $\rho_{(b, v)}(a, u)$ can be written also as

$$
u \Omega_{u, v}^{b}=u\left(u^{-1}\right)^{b}+\lambda_{u}(v) \quad\left(\Omega_{u, v}^{b}\right)^{-1} v=\rho_{v}\left(\left(\left(u^{-1}\right)^{b}\right)^{-1}\right)
$$

for all $(a, u),(b, v) \in S \times T$. We will use the two forms at the convenience of our computations.

Let us note that the maps associated to the double semidirect product of two skew left braces and that of two left cancellative left braces, applying Corollary 21 and Corollary 20, are automatically solutions. Since in general this does not happen, now we focus on sufficient conditions that allow for constructing new solutions through the double semidirect product of left semi-braces. We highlight that the condition 1 . in the following theorem can be generalized as one can see later in Remark 7, but our choice was dictated by the need to find examples in an easier way. 
Theorem 24. Let $S, T$ be left semi-braces and $B$ the double semidirect product of $S$ and $T$ via $\sigma$ and $\delta$. If $r_{S}$ and $r_{T}$ are solutions associated to $S$ and $T$, respectively, and the following are satisfied
1. $\left(u^{1}\right)^{a}=u^{a}$,
2. $1^{a}+u=1+u$,

for all $a \in S$ and $u \in T$, then the map $r_{B}$ associated to $B$ is a solution.

Proof. By Theorem 6, the map $r_{B}$ associated to $B$ is a solution if and only if the condition (2) is satisfied. If $(a, u),(b, v),(c, w) \in S \times T$, then we get

$$
\begin{aligned}
& \lambda_{(b, v)}(c, w)\left((1,1)+\rho_{(c, w)}(b, v)\right) \\
& =\lambda_{(b, v)}(c, w)\left((1,1)+\left(\left(\Omega_{v, w}^{c}\right)^{-1} \rho_{c}\left(v^{-1} b\right)\right),\left(\Omega_{v, w}^{c}\right)^{-1} w\right) \\
& =\left(\lambda_{b}\left({ }^{v} c\right), v \Omega_{v, w}^{c}\right)\left(1+{ }^{\left(\Omega_{v, w}^{c}\right)^{-1}} \rho_{c}\left(v^{-1} b\right), 1^{\left(\Omega_{v, w}^{c}\right)^{-1} \rho_{c}\left(v^{-1} b\right)}+\left(\Omega_{v, w}^{c}\right)^{-1} w\right) \\
& =\left(\lambda_{b}\left({ }^{v} c\right)^{v \Omega_{v, w}^{c}}\left(1+\left(\Omega_{v, w}^{c}\right)^{-1} \rho_{c}\left(v^{-1} b\right)\right), v \Omega_{v, w}^{c}\left(1^{\left(\Omega_{v, w}^{c}\right)^{-1} \rho_{c}\left(v^{-1} b\right)}+\left(\Omega_{v, w}^{c}\right)^{-1} w\right)\right) \\
& =\left(\lambda_{b}\left({ }^{v} c\right)\left(1+{ }^{v}\left(\rho_{c}\left(v^{-1} b\right)\right)\right), v \Omega_{v, w}^{c}\left(1^{\left(\Omega_{v, w}^{c}\right)^{-1} \rho_{c}\left(v^{-1} b\right)}+\left(\Omega_{v, w}^{c}\right)^{-1} w\right)\right),
\end{aligned}
$$

hence

$$
\begin{aligned}
& (a, u)+\lambda_{(b, v)}(c, w)\left((1,1)+\rho_{(c, w)}(b, v)\right) \\
& =\left(a+\lambda_{b}\left({ }^{v} c\right)\left(1+\rho_{v_{c}}(b)\right), u^{\lambda_{b}\left({ }^{v} c\right)\left(1+\rho_{v_{c}}(b)\right)}+v \Omega_{v, w}^{c}\left(1^{\left(\Omega_{v, w}^{c}\right)^{-1} \rho_{c}\left(v^{-1} b\right)}+\left(\Omega_{v, w}^{c}\right)^{-1} w\right)\right)
\end{aligned}
$$

and

$$
\begin{aligned}
(a, u)+(b, v)((1,1)+(c, w)) & =(a, u)+(b, v)\left(1+c, 1^{c}+w\right) \\
& =(a, u)+\left(b^{v}(1+c), v\left(1^{c}+w\right)\right) \\
& =\left(a+b\left(1+{ }^{v} c\right), u^{b\left(1+{ }^{v} c\right)}+v\left(1^{c}+w\right)\right) .
\end{aligned}
$$

Note that, by (2) in Theorem 6, since $r_{S}$ is a solution, the first components are equal. About the second components, we have that

$$
u^{\lambda_{b}\left({ }^{v} c\right)\left(1+\rho v_{c}(b)\right)}=\left(u^{1}\right)^{\lambda_{b}\left({ }^{v} c\right)\left(1+\rho v_{c}(b)\right)}=u^{1+\lambda_{b}\left({ }^{v} c\right)\left(1+\rho v_{c}(b)\right)} \overline{\overline{2}} u^{1+b\left(1+{ }^{v} c\right)}=\left(u^{1}\right)^{b\left(1+{ }^{v} c\right)}=u^{b\left(1+{ }^{v} c\right)}
$$


In addition,

$$
\begin{aligned}
& v \Omega_{v, w}^{c}\left(1^{\left(\Omega_{v, w}^{c}\right)^{-1} \rho_{c}\left(v^{-1} b\right)}+\left(\Omega_{v, w}^{c}\right)^{-1} w\right) \\
& =v \Omega_{v, w}^{c}\left(1+\left(\Omega_{v, w}^{c}\right)^{-1} w\right) \\
& =v\left(( v ^ { - 1 } ) ^ { c } \left(\left(\left(v^{-1}\right)^{c}\right)^{-1} \Omega_{v, w}^{c}\left(1+\left(\Omega_{v, w}^{c}\right)^{-1} w\right)\right.\right. \\
& =v\left(v^{-1}\right)^{c} \lambda_{\left(\left(v^{-1}\right)^{c}\right)^{-1}}(w)\left(1+\rho_{w}\left(\left(\left(v^{-1}\right)^{c}\right)^{-1}\right)\right) \\
& =v\left(v^{-1}\right)^{c}\left(\left(v^{-1}\right)^{c}\right)^{-1}(1+w) \\
& =v(1+w) \\
& =v\left(1^{c}+w\right) \text {. }
\end{aligned}
$$

Therefore, the claim follows.

Remark 6. Let us observe that, if $S$ and $T$ are left cancellative left semi-brace, then conditions 1 . and 2. of the previous theorem are satisfied. Indeed, if $a \in S$ and $u \in T$, since 1 is a left identity, $\left(u^{1}\right)^{a}=u^{1+a}=u^{a}$. Moreover, since $1^{a}$ is still an idempotent of $(T,+)$, it follows that $1^{a}+u=u=1+u$, hence the condition 2 . is satisfied.

Remark 7. The condition 1 . in the Theorem 24 can be replaced with the more general one

$$
u^{a b}=\left(u^{a}\right)^{\lambda_{a}(b)}
$$

for all $a, b \in S$ and $u \in T$. Indeed, if $a \in S$, since $1+a=1(1+a)=1+\lambda_{1}(a)$, we obtain that

$$
\left(u^{1}\right)^{a}=u^{1+a}=u^{1+\lambda_{1}(a)}=\left(u^{1}\right)^{\lambda_{1}(a)} \underset{\left(1^{\prime} .\right)}{=} u^{1 \cdot a}=u^{a}
$$

hence 1 . is satisfied. We also underline that the condition $1^{\prime}$. holds also for left cancellative left semi-braces. Indeed, in this case, if $a, b \in S$ we have that $a b=a(1+b)=a+\lambda_{a}(b)$, and so

$$
u^{a b}=u^{a+\lambda_{a}(b)}=\left(u^{a}\right)^{\lambda_{a}(b)},
$$

for every $u \in T$.

In the following, we construct two left inverse semi-braces in which the additive structure is a rectangular band.

Example 15. Let $S$ be the left semi-brace with $(S,+)$ the left zero semigroup and $(S, \cdot)$ a group with identity 1 , and $T$ the brace with additive group $(\mathbb{Z},+)$ and multiplication $\cdot$ given by $u \cdot v:=u+(-1)^{u} v$, for all $u, v \in \mathbb{Z}$ (see [12, Example 2]), where it is used the juxtaposition to denote the usual multiplication in $\mathbb{Z}$. Thus, the maps $r_{S}(a, b)=(1, a b)$ and $r_{T}(u, v)=\left((-1)^{u} v,(-1)^{v} u\right)$ are the solutions associated to $S$ and $T$, respectively. Moreover, let $\delta$ be the anti-homomorphism from $(S,+)$ into the endomorphism semigroup of $(T,+)$ given by $\delta(a)(u)=0$, for 
all $a \in S$ and $u \in T$. Note that with these assumptions the conditions 1 . and 2. of Theorem 24 are trivially satisfied. It is a routine computation to check that the condition (18) holds independently by the choice of the map $\sigma$. Now, we consider two maps $\sigma$ to obtain two distinct semi-braces which are double semidirect products of $S$ and $T$.

- If $\sigma(u)=\operatorname{id}_{S}$, for every $u \in T$, we get that $S \times T$ is the double semidirect product of $S$ and $T$ via $\sigma$ and $\delta$ endowed with

$$
(a, u)+(b, v)=(a, v) \quad(a, u)(b, v)=\left(a b, u+(-1)^{u} v\right)
$$

for all $(a, u),(b, v) \in S \times T$, which we denote by $B$. By Theorem 24 the map $r_{B}$ associated to $B$ given by

$$
\left.r_{B}((a, u),(b, v))=\left(\left(1, u+(-1)^{u} v\right)\right),(a b, 0)\right)
$$

is a solution. In addition, $r_{B}$ is idempotent.

- Consider $\sigma(u)=\operatorname{id}_{S}$, if $u$ is even, instead $\sigma(u)=\iota$, if $u$ is odd, where $\iota: \mathbb{Z} \rightarrow \mathbb{Z}$ is the map given by $\iota(u)=-u$, for every $u \in \mathbb{Z}$. Then, $S \times T$ is the double semidirect product of $S$ and $T$ via $\sigma$ and $\delta$ endowed with

$$
(a, u)+(b, v)=(a, v) \quad(a, u)(b, v)=\left(a^{u} b, u+(-1)^{u} v\right)
$$

for all $(a, u),(b, v) \in S \times T$, which we denote by $B$. By Theorem 24 the map $r_{B}$ associated to $B$ given by

$$
\left.r_{B}((a, u),(b, v))=\left(\left(1, u+(-1)^{u} v\right)\right),\left(v^{-1}\left(u^{-1} a b\right), 0\right)\right)
$$

is a solution. In addition, $r_{B}$ is idempotent.

We remark that the two solutions above lie in the class of solutions associated to left semi-braces having $\rho$ as an anti-homomorphism.

The following is an example of completely simple left semi-brace. In particular, by [17, Theorem 3], the additive structure $(S \times T,+)$ is a rectangular group.

Example 16. Let $S$ be the left semi-brace with additive group $(\mathbb{Z},+)$ and multiplication $\cdot$ given by $a \cdot b=a+(-1)^{a} b$, for all $a, b \in \mathbb{Z}$, where it is used the juxtaposition to denote the usual multiplication in $\mathbb{Z}$. Let $T$ be the left semi-brace with multiplicative group $\left(\operatorname{Sym}_{3}, \circ\right)$, where $\circ$ is the usual composition of maps, and addition + given by $u+v:=$ $v \circ g\left(v^{-1}\right) \circ u$, for all $u, v \in \mathrm{Sym}_{3}$, with $g$ the idempotent endomorphism of $\mathrm{Sym}_{3}$ defined by

$$
g(u)= \begin{cases}(12) & \text { if } u \text { is odd } \\ \operatorname{id}_{\mathrm{Sym}_{3}} & \text { otherwise }\end{cases}
$$

for every $u \in \operatorname{Sym}_{3}$ (cf. [17, Example 5-2]). If $s: \operatorname{Sym}_{3} \rightarrow \mathbb{N}_{0}$ is the map defined by $s(u)=1$, if $u$ is an odd permutation, and $s(u)=0$, otherwise, we can briefly write $g(u)=(12)^{s(u)}$, for every $u \in \mathrm{Sym}_{3}$. Now, set 
$\delta(a)=g$, for every $a \in \mathbb{Z}$, since $g$ is an idempotent endomorphism, we have that $\delta$ in an anti-homomorphism from $(\mathbb{Z},+)$ into the endomorphism monoid of $\left(\mathrm{Sym}_{3},+\right)$. Moreover, if $a, b \in \mathbb{Z}$ and $u, v \in \mathrm{Sym}_{3}$, we get

$$
\begin{aligned}
(u \circ v)^{\lambda_{a}\left({ }^{u} b\right)} & +u \circ\left(\left(u^{-1}\right)^{b}+w\right)=g(u \circ v)+u \circ\left(g\left(u^{-1}\right)+w\right) \\
& =g(u \circ v)+u \circ w \circ g\left(w^{-1}\right) \circ g\left(u^{-1}\right) \\
& =u \circ w \circ g\left(w^{-1}\right) \circ g\left(u^{-1}\right) \circ g\left(u \circ w \circ g\left(w^{-1}\right) \circ g\left(u^{-1}\right)\right)^{-1} \circ g(u \circ v) \\
& =u \circ w \circ g\left(w^{-1}\right) \circ g(v)=u \circ\left(v^{b}+w\right),
\end{aligned}
$$

hence (18) holds, independently by the choice of the map $\sigma$. Just to give an example, let us define $\sigma: \operatorname{Sym}_{3} \rightarrow \operatorname{Aut}_{(S)}$ the homomorphism from $\left(\mathrm{Sym}_{3}, \circ\right)$ into the automorphism group of the left semi-brace $S$ given by

$$
\sigma(u)= \begin{cases}\iota & \text { if } u \text { is odd }=\iota s(u), \\ \operatorname{id}_{\mathbb{Z}} & \text { otherwise }\end{cases}
$$

for every $u \in \operatorname{Sym}_{3}$, where $\iota: \mathbb{Z} \rightarrow \mathbb{Z}$ is the map defined by $\iota(a)=-a$, for every $a \in \mathbb{Z}$. Therefore, we obtain a double semidirect product on $S \times T$ via $\sigma$ and $\delta$ which we denote by $B$. Now, if we consider the maps $r_{S}$ and $r_{T}$ associated to $S$ and $T$ are respectively given by $r_{S}(a, b)=\left((-1)^{a} b,(-1)^{b} a\right)$ and $r_{T}(u, v)=\left(u \circ v \circ(12)^{s(v)} \circ u^{-1}, u \circ(12)^{s(v)}\right)$, they are solutions and the conditions 1. and 2. of Theorem 24 are trivially satisfied. Hence, the map $r_{B}$ associated to the left semi-brace $B$ having components

$$
\begin{aligned}
& \lambda_{(a, u)}(b, v)=\left((-1)^{a+s(u)} b, u \circ v \circ(12)^{s(u \circ v)}\right) \\
& \rho_{(b, v)}(a, u)=\left((12)^{s(u \circ v) \circ v^{-1}}\left((-1)^{b+s(u)} a\right),(12)^{s(u \circ v)}\right),
\end{aligned}
$$

for all $(a, u),(b, v) \in S \times T$, is a solution. Furthermore, one can check that the map $\rho$ is an anti-homomorphism from $(B, \cdot)$ into the monoid of the maps from $B$ into itself, hence, $r_{B}$ is a solution on $B$ and, by [35, Proposition 2.14], $(B,+, \cdot)$ is a completely simple left semi-brace.

Starting from the semidirect product of the inverse semigroups $S$ and $T$ via $\sigma$ in Example 9 , we show how to obtain five examples of double semidirect products by choosing all the possible maps $\delta$.

Example 17. Let $S$ and $T$ be the inverse semigroups in Example 9 , set $a+b=b \cdot 1=1$, for all $a, b \in S$, and $u+v=u v$, for all $u, v \in T$, then $S$ and $T$ are the left inverse semi-braces in Example 3 and Examples 4 respectively. Recall that the solution $r_{S}$ associated to $S$ is given by $r_{S}(a, b)=(1,1)$ (see Example7) and, since $u^{-1}=u$, for every $u \in T$, the solution $r_{T}$ associated to $T$ is given by $r_{T}(u, v)=\left(u^{2} v, u v^{2}\right)$ (see Examples 8). Now, recall that if $\tau$ is the automorphism of $S$ given by $\tau:=(x y)$, then the map $\sigma: T \rightarrow \operatorname{Aut}(S)$ given by $\sigma(1)=\sigma(x)=\operatorname{id}_{S}$, and $\sigma(y)=\tau$ is a homomorphism from $(T, \cdot)$ into the automorphism group of the left inverse semi-brace $S$. One can check that there exist five endomorphisms $\varphi$ of $(T,+)$ and, in particular, they are all idempotent. Moreover, note that to obtain $\delta$ an anti-homomorphism we have to choose $\delta(a)=\varphi$, for every $a \in S$, with $\varphi \in \operatorname{End}(T,+)$. In each of these cases, it 
is easy to prove that condition (18) holds. Thus, for every fixed $\delta$, we have that $B:=S \times T$ is the double semidirect product of $S$ and $T$ via $\sigma$ and $\delta$ with respect to

$$
(a, u)+(b, v)=(1, \varphi(u) v) \quad(a, u)(b, v)=\left(a^{u} b, u v\right)
$$

for all $(a, u),(b, v) \in S \times T$. Now, distinguishing the various cases, we analyze that the maps $r_{B}$ associated to each semi-brace $B$.

- If $\varphi=\mathrm{id}_{T}$ :

$$
r_{B}((a, u),(b, v))=\left(\left(1, u^{2} v\right),\left(1, u v^{2}\right)\right)
$$

which clearly is a solution, since it is the semidirect product of $r_{S}$ and $r_{T}$. In particular, by [16, Proposition $10]$, it is a cubic solution.

- If $\varphi=k_{1}$, the constant map from $T$ into itself of value 1 :

$$
r_{B}((a, u),(b, v))=\left((1, u v),\left(1, v^{2}\right)\right)
$$

which is an idempotent solution.

- If $\varphi=k_{x}$, the constant map from $T$ into itself of value $x$ :

$$
r_{B}((a, u),(b, v))=\left((1, x u v),\left(1, x v^{2}\right)\right)
$$

which is an idempotent solution.

- If $\varphi$ is the map from $T$ into itself defined by $\varphi(1)=1$ and $\varphi(x)=\varphi(y)=x$ :

$$
r_{B}((a, u),(b, v))=\left((1, \varphi(u) u v),\left(1, \varphi(u) v^{2}\right)\right)
$$

is a solution and it satisfies $r_{B}^{5}=r_{B}^{3}$.

- If $\varphi$ the map from $T$ into itself defined by $\varphi(1)=\varphi(x)=x$ and $\varphi(y)=y$ :

$$
r_{B}((a, u),(b, v))=\left((1, \varphi(u) u v),\left(1, \varphi(u) v^{2}\right)\right)
$$

is not a solution. Indeed, if $a, b, c$ are arbitrary elements of $S, u=w=1$, and $v=y$, one can check that the braid relation is not satisfied.

In light of the previous example, it arises the following question.

Question 25. Let $S, T$ be left inverse semi-braces having solutions $r_{S}$ and $r_{T}$ and $B$ the double semidirect product of $S$ and $T$ via $\sigma$ and $\delta$. Under which assumptions the map $r_{B}$ is a solution? 


\section{The asymmetric product of left inverse semi-braces}

This section aims to introduce a generalization of the asymmetric product of left cancellative left semi-braces given in [13], involving left inverse semi-braces. We highlight that, in general, this construction does not include the double semidirect product. Moreover, we provide sufficient conditions to obtain solutions.

To present the asymmetric product of left inverse semi-braces, we need the notion of cocycle on semigroups. In particular, the following definition is inspired to that used for groups in the context of Schreier's extension (see in [32, Theorem 15.1.1]).

Definition 26. Let $(S,+)$ and $(T,+)$ be two semigroups (not necessarily commutative) and $\delta: S \rightarrow$ End $(T)$ a map from $S$ into the endomorphism semigroup of $T$. Set $u^{a}:=\delta(a)(u)$, for all $a \in S$ and $u \in T$, a map $\mathfrak{b}: S \times S \rightarrow T$ is called a $\delta$-cocycle if

$$
\mathfrak{b}(a+b, c)+\mathfrak{b}(a, b)^{c}+\left(u^{b}\right)^{c}+v^{c}=\mathfrak{b}(a, b+c)+u^{b+c}+\mathfrak{b}(b, c)+v^{c}
$$

holds, for all $a, b, c \in S$ and $u, v \in T$.

The notion of cocycle was already recovered in [13, p. 173] for left cancellative left semi-braces. Specifically, the concept of $\delta$-cocycle in Definition 26 involves entirely the additive structures of $S$ and $T$, hence it is not a simple readjustment of that introduced in [13]. Let us compare into detail the two definitions.

Remark 8. Let $(S,+),(T,+)$ be right groups, $S:=H+E$ and $T:=N+F$ where $H \cap E=\left\{1_{S}\right\}, N \cap F=\left\{1_{T}\right\}$, $E$ and $F$ are the sets of idempotents of $S$ and $T$, respectively, and $H=S+1$ and $N=T+1$. Let $\alpha: H \rightarrow \operatorname{Aut}(N)$ and $\mathfrak{c}: H \times H \rightarrow N$ be maps such that $(\alpha, \mathfrak{c})$ is a cocycle as in [13, p. 11]. Define the map $\mathfrak{b}: S \times S \rightarrow T$ given by

$$
\mathfrak{b}\left(h_{1}+e_{1}, h_{2}+e_{2}\right):=\mathfrak{c}\left(h_{1}, h_{2}\right)
$$

for every $\left(h_{1}+e_{1}, h_{2}+e_{2}\right) \in S \times S$, with $h_{1}, h_{2} \in H$ and $e_{1}, e_{2} \in E$, and $\delta: S \rightarrow$ End $(T)$ the map given by

$$
\delta(h+e)(n+f)=(n+f)^{h+e}:=n^{h}+f
$$

for all $h+e \in S$ and $n+f \in T$. If $(a, u),(b, v) \in S \times T$, with $(a, u)=\left(h_{1}+e_{1}, n_{1}+f_{1}\right)$ and $(b, v)=\left(h_{2}+e_{2}, n_{2}+f_{2}\right)$, since $e_{1}$ and $f_{1}$ are idempotents, then we have that

$$
\begin{aligned}
(a, u)+(b, v) & =\left(h_{1}+e_{1}+h_{2}+e_{2}, \mathfrak{b}\left(h_{1}+e_{1}, h_{2}+e_{2}\right)+\left(n_{1}+f_{1}\right)^{\left(h_{2}+f_{2}\right)}+n_{2}+f_{2}\right) \\
& =\left(h_{1}+h_{2}+e_{2}, \mathfrak{c}\left(h_{1}, h_{2}\right)+n_{1}^{h_{2}}+f_{1}+n_{2}+f_{2}\right) \\
& =\left(h_{1}+h_{2}+e_{2}, \mathfrak{c}\left(h_{1}, h_{2}\right)+n_{1}^{h_{2}}+n_{2}+f_{2}\right)
\end{aligned}
$$


which is exactly the sum in [13, Theorem 12]. Now, recalling conditions 1. and 2. in [13, p. 173], namely,

$$
\begin{aligned}
& \left(n^{h_{1}}\right)^{h_{2}}=-\mathfrak{c}\left(h_{1}, h_{2}\right)+n^{h_{1}+h_{2}}+\mathfrak{c}\left(h_{1}, h_{2}\right) \\
& \mathfrak{c}\left(h_{1}+h_{2}, h_{3}\right)+\mathfrak{c}\left(h_{1}, h_{2}\right)^{h_{3}}=\mathfrak{c}\left(h_{1}, h_{2}+h_{3}\right)+\mathfrak{c}\left(h_{2}, h_{3}\right),
\end{aligned}
$$

for all $h_{1}, h_{2}, h_{3} \in H$ and $n \in N$, if $a=h_{1}+e_{1}, b=h_{2}+e_{2}, c=h_{3}+e_{3}$ are elements of $S$, and $u=n_{1}+f_{1}$, $v=n_{2}+f_{2}$ are elements of $T$, it follows that

$$
\begin{aligned}
& \mathfrak{b}(a+b, c)+\mathfrak{b}(a, b)^{c}+\left(u^{b}\right)^{c}+v^{c} \\
& =\mathfrak{c}\left(h_{1}+h_{2}, h_{3}\right)+\mathfrak{c}\left(h_{1}, h_{2}\right)^{h_{3}}+\left(n_{1}^{h_{2}}\right)^{h_{3}}+f_{1}+v^{c} \\
& =\mathfrak{c}\left(h_{1}+h_{2}, h_{3}\right)+\mathfrak{c}\left(h_{1}, h_{2}\right)^{h_{3}}+\left(n^{h_{2}}\right)^{h_{3}}+v^{c} \\
& =\mathfrak{c}\left(h_{1}, h_{2}+h_{3}\right)+\mathfrak{c}\left(h_{2}, h_{3}\right)+\left(n^{h_{2}}\right)^{h_{3}}+v^{c} \\
& =\mathfrak{c}\left(h_{1}, h_{2}+h_{3}\right)+n^{h_{2}+h_{3}}+f_{1}+\mathfrak{c}\left(h_{2}, h_{3}\right)+v^{c} \\
& =\mathfrak{b}(a, b+c)+u^{b+c}+\mathfrak{b}(b, c)+v^{c},
\end{aligned}
$$

i.e., $\mathfrak{b}$ is a $\delta$-cocycle.

In the following theorem we provide the construction of the asymmetric product of left inverse semi-braces.

Theorem 27. Let $S, T$ be left inverse semi-braces, $\sigma: T \rightarrow \operatorname{Aut}(S)$ a homomorphism from $(T, \cdot)$ into the automorphism group of the left inverse semi-brace $S$, with ${ }^{u} a:=\sigma(u)(a)$, for all $a \in S$ and $u \in T$, and $\delta: S \rightarrow$ End (T) $a$ map from $S$ into the endomorphism semigroup of $(T,+)$. If $\mathfrak{b}$ is a $\delta$-cocycle such that

$$
\mathfrak{b}\left(a^{u} b, \lambda_{a}\left({ }^{u} c\right)\right)+(u v)^{\lambda_{a}\left({ }^{u} c\right)}+u\left(\mathfrak{b}\left(u^{-1}\left(a^{-1}\right), c\right)+\left(u^{-1}\right)^{c}\right)=u\left(\mathfrak{b}(b, c)+v^{c}\right)
$$

holds, for all $a, b, c \in S$ and $u, v \in T$, then $S \times T$ with respect to

$$
\begin{aligned}
(a, u)+(b, v) & :=\left(a+b, \mathfrak{b}(a, b)+u^{b}+v\right) \\
(a, u)(b, v) & :=\left(a^{u} b, u v\right)
\end{aligned}
$$

for all $(a, u),(b, v) \in S \times T$, is a inverse semi-brace. We call such a left inverse semi-brace the asymmetric product of $S$ and $T$ via $\sigma, \delta$, and $\mathfrak{b}$.

Proof. Initially, by applying (19), it is a routine computation to see that $(S \times T,+)$ is a semigroup. Moreover, by Theorem 16, $(S \times T, \cdot)$ is an inverse semigroup. Now, to get the claim we have only to check that (1) holds. If $(a, u),(b, v),(c, w) \in S \times T$, we obtain

$$
(a, u)((b, v)+(c, w))=\left(a^{u}(b+c), u\left(\mathfrak{b}(b, c)+v^{c}+w\right)\right)
$$


and

$$
\begin{aligned}
& (a, u)(b, v)+(a, u)\left((a, u)^{-1}+(c, w)\right) \\
& =\left(a^{u} b, u v\right)+(a, u)\left(u^{-1} a^{-1}+c, \mathfrak{b}\left(u^{-1} a^{-1}, c\right)+\left(u^{-1}\right)^{c}+w\right) \\
& =\left(a^{u} b, u v\right)+\left(a^{u}\left(u^{-1} a^{-1}+c\right), u\left(\mathfrak{b}\left(u^{-1} a^{-1}, c\right)+\left(u^{-1}\right)^{c}+w\right)\right) \\
& =\left(a^{u} b+a\left({ }^{u u^{-1}} a^{-1}+{ }^{u} c\right), \mathfrak{b}\left(a^{u} b, a\left(u^{-1} a^{-1}+{ }^{u} c\right)\right)+(u v)^{a\left(u^{-1} a^{-1}+{ }^{u} c\right)}\right. \\
& \left.\quad+u\left(\mathfrak{b}\left({ }^{u^{-1}}\left(a^{-1}\right), c\right)+\left(u^{-1}\right)^{c}+w\right)\right) \\
& =\left(a^{u} b+\lambda_{a}\left({ }^{u} c\right), \mathfrak{b}\left(a^{u} b, \lambda_{a}\left({ }^{u} c\right)\right)+(u v)^{\lambda_{a}\left({ }^{u} c\right)}+u\left(\mathfrak{b}\left(u^{-1}\left(a^{-1}\right), c\right)+\left(u^{-1}\right)^{c}+w\right)\right)
\end{aligned}
$$

where in the last equality we apply (16). Now, we observe that

$$
a^{u} b+\lambda_{a}\left({ }^{u} c\right)=a\left({ }^{u} b+{ }^{u} c\right)=a^{u}(b+c)
$$

and

$$
\begin{aligned}
\mathfrak{b} & \left(a^{u} b, \lambda_{a}\left({ }^{u} c\right)\right)+(u v)^{\lambda_{a}\left({ }^{u} c\right)}+u\left(\mathfrak{b}\left(u^{-1}\left(a^{-1}\right), c\right)+\left(u^{-1}\right)^{c}+w\right) \\
& =\mathfrak{b}\left(a^{u} b, \lambda_{a}\left({ }^{u} c\right)\right)+(u v)^{\lambda_{a}\left({ }^{u} c\right)}+u\left(\mathfrak{b}\left(u^{-1}\left(a^{-1}\right), c\right)+\left(u^{-1}\right)^{c}\right)+u\left(u^{-1}+w\right) \\
& =u\left(\mathfrak{b}(b, c)+v^{c}\right)+u\left(u^{-1}+w\right) \\
& =u\left(\mathfrak{b}(b, c)+v^{c}+w\right) .
\end{aligned}
$$

Therefore, $S \times T$ is a left inverse semi-brace.

Let us compare [13, Theorem 12] with Theorem 27, in the specific case of two left cancellative left semi-braces.

Remark 9. Let $(S,+),(T,+)$ be right groups, $S:=H+E$ and $T:=N+F$ where $H \cap E=\{1\}, N \cap F=\{1\}, E$ and $F$ are the sets of idempotents of $S$ and $T$, respectively, and $H=S+1$ and $N=T+1$. Let us consider the maps $\mathfrak{b}$ and $\delta$ defined in Remark 8 and $a=h_{1}+e_{1}, b=h_{2}+e_{2}, c=h_{3}+e_{3} \in S$, and $u=n_{1}+f_{1}, v=n_{2}+f_{2} \in T$. Recalling that in any left cancellative left semi-brace it holds $x \cdot y=x+\lambda_{x}(y)$, by [13, Proposition 7-1.], we obtain that

$$
\begin{aligned}
a^{u} b & =h_{1}+e_{1}+\lambda_{h_{1}+e_{1}}\left(\left(n_{1}+f_{1}\right)\left(h_{2}+e_{2}\right)\right)=h_{1}+e_{1}+\lambda_{h_{1}+e_{1}}\left(\left(n_{1}+f_{1}\right)\left(h_{2}\right)\right)+\lambda_{h_{1}+e_{1}}\left(\left(n_{1}+f_{1}\right)\left(e_{2}\right)\right) \\
& =\underbrace{\left(h_{1}+e_{1}\right)\left(\left(n_{1}+f_{1}\right)\left(h_{2}\right)\right)+1}_{H}+\underbrace{\lambda_{h_{1}+e_{1}}\left(\left(n_{1}+f_{1}\right)\right.}_{E} e_{2})
\end{aligned}
$$

and

$$
\lambda_{a}\left({ }^{u} c\right)=\underbrace{\lambda_{h_{1}+e_{1}}\left({ }^{\left(n_{1}+f_{1}\right)} h_{3}\right)+1}_{H}+\underbrace{\lambda_{h_{1}+e_{1}}\left(e_{3}\right)}_{E}
$$


and, by [13, Lemma 11-1.],

$$
a^{-1}=\left(h_{1}+e_{1}\right)^{-1} e_{1}+\left(h_{1}+e_{1}\right)^{-1} h_{1}=\underbrace{\rho_{e_{1}}\left(h_{1}^{-1}\right)}_{H}+\underbrace{\left(\lambda_{h_{1}^{-1}}\left(e_{1}\right)\right)^{-1}}_{E} .
$$

Analogously, $u^{-1}=\underbrace{\rho_{f_{1}}\left(n_{1}^{-1}\right)}_{N}+\underbrace{\left(\lambda_{n_{1}^{-1}}\left(f_{1}\right)\right)^{-1}}_{F}$. Moreover, $u v=\underbrace{\left(n_{1}+f_{1}\right) n_{2}+1}_{N}+\underbrace{\lambda_{n_{1}+f_{1}}\left(f_{2}\right)}_{F}$, hence we get

$$
(u v)^{\lambda_{a}\left({ }^{u} c\right)}=\underbrace{\left(\left(n_{1}+f_{1}\right) n_{2}+1\right)^{\lambda_{h_{1}+e_{1}}\left({ }^{\left(n_{1}+f_{1}\right)} h_{3}+1\right)}}_{N}+\underbrace{\lambda_{n_{1}+f_{1}}\left(f_{2}\right)}_{F} .
$$

Since $\lambda_{n_{1}+f_{1}}\left(f_{2}\right) \in F$, by condition (5) of [13, Theorem 12] and by recalling that $\mathfrak{b}\left(h_{1}+e_{1}, h_{2}+e_{2}\right)=\mathfrak{c}\left(h_{1}, h_{2}\right)$, for every $\left(h_{1}+e_{1}, h_{2}+e_{2}\right) \in S \times S$, it follows that

$$
\begin{aligned}
\mathfrak{b} & \left(a^{u} b, \lambda_{a}\left({ }^{u} c\right)\right)+(u v)^{\lambda_{a}\left({ }^{u} c\right)}+u\left(\mathfrak{b}\left(u^{-1}\left(a^{-1}\right), c\right)+\left(u^{-1}\right)^{c}\right) \\
= & \mathfrak{c}\left(\left(h_{1}+e_{1}\right)^{n_{1}+f_{1}} h_{2}, \lambda_{h_{1}+e_{1}}\left({ }^{\left(n_{1}+f_{1}\right)} h_{3}+1\right)+1\right) \\
& +\left(\left(n_{1}+f_{1}\right) n_{2}+1\right)^{\lambda_{h_{1}+e_{1}}\left({ }^{\left(n_{1}+f_{1}\right)} h_{3}+1\right)}+\lambda_{n_{1}+f_{1}}\left(f_{2}\right) \\
& +\left(n_{1}+f_{1}\right)\left(\mathfrak{c}\left({ }^{{ }^{-1}} \rho_{e_{1}}\left(h_{1}^{-1}\right), h_{3}\right)+\rho_{f_{1}}\left(n_{1}^{-1}\right)^{h_{3}}\right) \\
= & \mathfrak{c}\left(\left(h_{1}+e_{1}\right){ }^{n_{1}+f_{1}} h_{2}+1, \lambda_{h_{1}+e_{1}}\left({ }^{n_{1}+f_{1}} h_{3}\right)+1\right) \\
& +\left(\left(n_{1}+f_{1}\right) n_{2}+1\right)^{\lambda_{h_{1}+e_{1}}\left({ }^{\left(n_{1}+f_{1}\right)} h_{3}+1\right)} \\
& +\left(n_{1}+f_{1}\right)\left(\mathfrak{c}\left(\left(n_{1}+f_{1}\right)^{-1} \rho_{e_{1}}\left(h_{1}^{-1}\right), h_{3}\right)+\rho_{f_{1}}\left(n_{1}^{-1}\right)^{h_{3}}\right) \\
= & \left(n_{1}+f_{1}\right)\left(\mathfrak{c}\left(h_{2}, h_{3}\right)+n_{2}^{h_{3}}\right) \\
= & u\left(\mathfrak{b}(b, c)+v^{c}\right),
\end{aligned}
$$

namely, condition (20) is satisfied.

In the following theorem we give the map associated to any asymmetric product of left inverse semi-braces. We omit the proof since it is similar to that in Proposition 23. We recall the notation already adopted, that is $\Omega_{u, v}^{a}:=$ $\left(u^{-1}\right)^{a}+v$, for all $a \in S$ and $u, v \in T$.

Proposition 28. Let $S, T$ be left inverse semi-braces and $B$ the asymmetric product of $S$ and $T$ via $\sigma$ and $\delta$ and $\mathfrak{b}$. Then, the map $r_{B}$ associated to $B$ is given by

$$
\begin{aligned}
r_{B}((a, u),(b, v))= & \left(\left(\lambda_{a}\left({ }^{u} b\right), u\left(\mathfrak{b}\left(u^{-1} a^{-1}, b\right)+\Omega_{u, v}^{b}\right)\right),\right. \\
& \left.\left(\mathfrak{b}\left(u^{-1} a^{-1}, b\right)+\left(\Omega_{u, v}^{b}\right)^{-1} u^{-1} \rho_{u b}(a),\left(\mathfrak{b}\left(u^{-1} a^{-1}, b\right)+\Omega_{u, v}^{b}\right)^{-1} v\right)\right),
\end{aligned}
$$

for all $(a, u),(b, v) \in S \times T$. 
Note that the second component of $\rho_{(b, v)}(a, u)$ can be written also as

$$
\left(\mathfrak{b}\left(u^{-1} a^{-1}, b\right)+\Omega_{u, v}^{b}\right)^{-1} v=\rho_{v}\left(\left(\mathfrak{b}\left(u^{-1} a^{-1}, b\right)+\left(u^{-1}\right)^{b}\right)^{-1}\right)
$$

for all $(a, u),(b, v) \in S \times T$.

Now, we focus on sufficient conditions that allow for constructing new solutions through the asymmetric product of left semi-braces.

Theorem 29. Let $S$, $T$ be left semi-braces and $B$ the asymmetric product of $S$ and $T$ via $\sigma$, $\delta$, and $\mathfrak{b}$. If $r_{S}$ and $r_{T}$ are solutions associated to $S$ and $T$, respectively, and the following are satisfied

$$
\begin{aligned}
& \text { 1. }\left(u^{1}\right)^{a}=u^{a}, \\
& \text { 2. } \mathfrak{b}(1, a)+u=1+u, \\
& \text { 3. } \mathfrak{b}(a, 1+b)=\mathfrak{b}(a, b),
\end{aligned}
$$

for all $a, b \in S$ and $u \in T$, then the map $r_{B}$ associated to $B$ is a solution.

Proof. To prove that the map $r_{B}$ is a solution, we check that the condition (2) in Theorem 6 is satisfied. If $(a, u),(b, v),(c, w) \in S \times T$, by 2 ., we get

$$
\begin{aligned}
& (a, u)+(b, v)((1,1)+(c, w)) \\
& =\left(a+b\left(1+{ }^{v} c\right), \mathfrak{b}\left(a, b\left(1+{ }^{v} c\right)\right)+u^{b\left(1+{ }^{v} c\right)}+v\left(\mathfrak{b}(1, c)+1^{c}+w\right)\right) \\
& =\left(a+b\left(1+{ }^{v} c\right), \mathfrak{b}\left(a, b\left(1+{ }^{v} c\right)\right)+u^{b\left(1+{ }^{v} c\right)}+v(1+w)\right)
\end{aligned}
$$

since $1^{c}$ is idempotent and by Proposition 5 it is a middle unit. Moreover,

$$
\begin{aligned}
& (a, u)+\lambda_{(b, v)}(c, w)\left((1,1)+\rho_{(c, w)}(b, v)\right) \\
& =\left(a+\lambda_{b}\left({ }^{v} c\right)\left(1+\rho_{v_{c}}(b)\right), \mathfrak{b}\left(a, \lambda_{b}\left({ }^{v} c\right)\left(1+\rho_{v_{c}}(b)\right)\right)+u^{\lambda_{b}\left({ }^{v} c\right)\left(1+\rho v_{c}(b)\right)}\right. \\
& +v\left(\mathfrak{b}\left(v^{-1} b^{-1}, c\right)+\Omega_{v, w}^{c}\right)\left(\mathfrak{b}\left(1,\left(\mathfrak{b}\left(v^{-1} b^{-1}, c\right)+\Omega_{v, w}^{c}\right)^{-1} v^{-1} \rho_{v} c(b)\right)\right. \\
& \left.+1\left(\mathfrak{b}^{\left.\left.-1 v^{-1}, c\right)+\Omega_{v, w}^{c}\right)^{-1} v^{-1} \rho v_{c}(b)}+\left(\mathfrak{b}\left(v^{-1} b^{-1}, c\right)+\Omega_{v, w}^{c}\right)^{-1} w\right)\right) \text {. }
\end{aligned}
$$

Note that the first components are equal since $r_{S}$ is a solution. In addition,

$$
\begin{array}{rlrl}
\mathfrak{b}\left(a, \lambda_{b}\left({ }^{v} c\right)\left(1+\rho^{v_{c}}(b)\right)\right) & =\mathfrak{b}\left(a, 1+\lambda_{b}\left({ }^{v} c\right)\left(1+\rho^{v_{c}}(b)\right)\right) & & \text { by } 3 . \\
& =\mathfrak{b}\left(a, b\left(1+{ }^{v} c\right)\right) & \text { by (2) }
\end{array}
$$


Furthermore, since $1^{\left(\mathfrak{b}\left(v^{-1} b^{-1}, c\right)+\Omega_{v}^{c}, w\right)^{-1} v^{-1}} \rho_{v_{c}}(b)$ is an idempotent it is a middle unit and so, by 2 , we get

$$
\begin{aligned}
& \mathfrak{b}\left(1,\left(\mathfrak{b}\left(v^{-1} b^{-1}, c\right)+\Omega_{v, w}^{c}\right)^{-1} v^{-1} \rho_{v} c(b)\right)+\left(\mathfrak{b}\left(v^{-1} b^{-1}, c\right)+\Omega_{v, w}^{c}\right)^{-1} w \\
& =1+\left(\mathfrak{b}\left(v^{-1} b^{-1}, c\right)+\Omega_{v, w}^{c}\right)^{-1} w
\end{aligned}
$$

Moreover, by 1. and (2), it follows that $u^{\lambda_{b}\left({ }^{v} c\right)\left(1+\rho v_{c}(b)\right)}=u^{b\left(1+{ }^{v} c\right)}$, hence,

$$
\begin{aligned}
& u^{\lambda_{b}\left({ }^{v} c\right)\left(1+\rho v_{c}(b)\right)}+v\left(\mathfrak{b}\left(v^{-1} b^{-1}, c\right)+\Omega_{v, w}^{c}\right)\left(1+\left(\mathfrak{b}\left(v^{-1} b^{-1}, c\right)+\Omega_{v, w}^{c}\right)^{-1} w\right) \\
& =u^{b\left(1+{ }^{v} c\right)}+v\left(\mathfrak{b}\left(v^{-1} b^{-1}, c\right)+\left(v^{-1}\right)^{c}\right) \text {. } \\
& \cdot \lambda_{\left(\mathfrak{b}\left(v^{-1} b^{-1}, c\right)+\left(v^{-1}\right)^{c}\right)^{-1}}(w)\left(1+\rho_{w}\left(\left(\mathfrak{b}\left(v^{-1} b^{-1}, c\right)+\left(v^{-1}\right)^{c}\right)^{-1}\right)\right) \\
& =u^{b\left(1+{ }^{v} c\right)}+v\left(\mathfrak{b}\left(v^{-1} b^{-1}, c\right)+\left(v^{-1}\right)^{c}\right)\left(\mathfrak{b}\left(v^{-1} b^{-1}, c\right)+\left(v^{-1}\right)^{c}\right)^{-1}(1+w) \\
& =u^{b\left(1+{ }^{v} c\right)}+v(1+w) \text {. }
\end{aligned}
$$

Thus, the second components are equal. Therefore, the claim follows.

Remark 10. As observed in Remark 6 , if $S$ and $T$ are left cancellative left semi-braces, then conditions 1. and 3. of the previous theorem are satisfied. In addition, note that if $a \in S$ and $u \in T$, since $(1,1)$ is a left identity in $S \times T$, we have

$$
(a, 1+u)=(a, u)=(1,1)+(a, u)=\left(1+a, \mathfrak{b}(1, a)+1^{a}+u\right)=(a, \mathfrak{b}(1, a)+u)
$$

thus the condition 2. also holds.

Remark 11. Note that, similarly to Remark 7 , assuming that conditions 2. and 3. in the Theorem 29 are satisfied, instead of condition 1 . we may assume the more general one

$$
u^{a b}=\left(u^{a}\right)^{\lambda_{a}(b)}
$$

for all $a, b \in S$ and $u \in T$.

Example 18. Let $X$ be a set and $\cdot$ a binary operation on $X$ such that $(X, \cdot)$ is an abelian group. Thus, setting $a+b:=b f\left(b^{-1} a\right)$, for all $a, b \in X$, with $f$ an idempotent endomorphism of $(X, \cdot)$, we have that $S:=(X,+, \cdot)$ is a left semi-brace, see [17, Example 5, case 3]. Moreover, let $T:=(X,+, \cdot)$ be the left brace with the sum given by $u+v=u v$, for all $u, v \in X$.

Let us consider the map $\delta: S \rightarrow \operatorname{End}(T)$ such that $u^{a}=1$, for all $a \in S$ and $u \in T$ and $\mathfrak{b}: S \times S \rightarrow T$ the $\delta$-cocycle defined by $\mathfrak{b}(a, b)=a$, for all $a, b \in S$. Thus, if $\sigma: T \rightarrow \operatorname{Aut}(S)$ is the map given by ${ }^{u} a=a$, for all $a \in S$ and $u \in T$, it follows that (20) is trivially satisfied. Therefore, $B:=S \times T$ is the asymmetric product of $S$ and $T$ via $\sigma, \delta$, 
and $\mathfrak{b}$ endowed with

$$
(a, u)+(b, v)=(a+b, a+v) \quad \text { and } \quad(a, u)(b, v)=(a b, u v)
$$

for all $(a, u),(b, v) \in S \times T$. Now, the solutions $r_{S}$ and $r_{T}$ are given by $r_{S}(a, b)=\left(a b f\left(b^{-1} a^{-1}\right), f(a b)\right)$ and $r_{T}(u, v)=\left(v, v^{-1} u v\right)$, respectively. Since the conditions in Theorem 29 trivially hold, then we obtain that the map

$$
r_{B}((a, u),(b, v))=\left(\left(a b f\left(b^{-1} a^{-1}\right), u a^{-1} v\right),(f(a b), a)\right)
$$

is a solution. Furthermore, it holds $r_{B}^{3}=r_{B}^{2}$.

The following is a simple class of examples of asymmetric product of inverse semi-braces.

Example 19. Let $S$ be an arbitrary left inverse semi-brace and $T$ the left inverse semi-brace with $(T,+)$ the left zero semigroup and $(T, \cdot)$ an upper semilattice with join 1. Moreover, let $\sigma: T \rightarrow \operatorname{Aut}(S)$ be the homomorphism from $(T, \cdot)$ into the automorphism group of the left inverse semi-brace $S$ given by ${ }^{u} a=a$, for all $a \in S$ and $u \in T$, and $\delta: S \rightarrow \operatorname{End}(T)$ an arbitrary map from $S$ into the endomorphism monoid of $(T,+)$, and $\mathfrak{b}: S \times S \rightarrow T$ the $\delta$-cocycle defined $\mathfrak{b}(a, b)=1$, for all $a, b \in S$. Therefore, by Theorem 27, $S \times T$ is a left inverse semi-brace with

$$
(a, u)+(b, v)=(a+b, 1) \quad(a, u)(b, v)=(a b, u v)
$$

for all $(a, u),(b, v) \in S \times T$. Moreover, the map $r_{B}$ is given by

$$
r_{B}((a, u),(b, v))=\left(\left(\lambda_{a}(b), 1\right),\left(\rho_{b}(a), 1\right)\right)
$$

which is trivially a solution.

Finally, sufficient conditions to obtain that the map $r_{B}$ associated to an asymmetric product $B$ of two left inverse semi-braces $S$ and $T$ are not yet known to the authors. Thus, it arises the following question.

Question 30. Let $S, T$ be left inverse semi-braces having solutions $r_{S}$ and $r_{T}$ and $B$ the asymmetric product of $S$ and $T$ via $\sigma, \delta$, and a $\delta$-cocycle $\mathfrak{b}$. Under which assumptions is the map $r_{B}$ a solution?

\section{References}

[1] E. Acri, M. Bonatto, Skew braces of size $p q$, Comm. Algebra 48 (5) (2020) 1872-1881.

URL https://doi.org/10.1080/00927872.2019.1709480

[2] E. Acri, R. Lutowski, L. Vendramin, Retractability of solutions to the Yang-Baxter equation and $p$-nilpotency of skew braces, Internat. J. Algebra Comput. 30 (1) (2020) 91-115.

URL https://doi.org/10.1142/S0218196719500656 
[3] D. Bachiller, F. Cedó, E. Jespers, J. Okniński, Asymmetric product of left braces and simplicity; new solutions of the Yang-Baxter equation, Commun. Contemp. Math. 21 (8) (2019) 1850042, 30.

URL https://doi.org/10.1142/S0219199718500426

[4] R. J. Baxter, Partition function of the eight-vertex lattice model, Ann. Physics 70 (1972) 193-228.

URL https : //doi .org/10.1016/0003-4916(72)90335-1

[5] E. Campedel, A. Caranti, I. Del Corso, Hopf-Galois structures on extensions of degree $p^{2} q$ and skew braces of order $p^{2} q$ : the cyclic Sylow $p$-subgroup case, J. Algebra 556 (2020) 1165-1210.

URL https://doi.org/10.1016/j.jalgebra.2020.04.009

[6] M. Castelli, F. Catino, M. M. Miccoli, G. Pinto, Dynamical extensions of quasi-linear left cycle sets and the Yang-Baxter equation, J. Algebra Appl. 18 (11) (2019) 1950220, 16.

URL https : //doi.org/10.1142/s0219498819502207

[7] M. Castelli, F. Catino, G. Pinto, A new family of set-theoretic solutions of the Yang-Baxter equation, Comm. Algebra 46 (4) (2018) 1622-1629.

URL https://doi.org/10.1080/00927872.2017.1350700

[8] M. Castelli, F. Catino, G. Pinto, Indecomposable involutive set-theoretic solutions of the Yang-Baxter equation, J. Pure Appl. Algebra 223 (10) (2019) 4477-4493.

URL https://doi.org/10.1016/j.jpaa.2019.01.017

[9] M. Castelli, F. Catino, G. Pinto, About a question of Gateva-Ivanova and Cameron on square-free set-theoretic solutions of the Yang-Baxter equation, Comm. Algebra 48 (6) (2020) 2369-2381.

URL https://doi.org/10.1080/00927872.2020.1713328

[10] M. Castelli, F. Catino, P. Stefanelli, Left non-degenerate set-theoretic solutions of the Yang-Baxter equation and dynamical extensions of q-cycle sets, arXiv preprint arXiv:2001.10774.

URL https: //arxiv.org/abs/2001.10774

[11] M. Castelli, G. Pinto, W. Rump, On the indecomposable involutive set-theoretic solutions of the Yang-Baxter equation of prime-power size, Comm. Algebra 48 (5) (2020) 1941-1955.

URL https://doi.org/10.1080/00927872.2019.1710163

[12] F. Catino, I. Colazzo, P. Stefanelli, Regular subgroups of the affine group and asymmetric product of radical braces, J. Algebra 455 (2016) 164-182.

URL http://dx.doi.org/10.1016/j.jalgebra.2016.01.038

[13] F. Catino, I. Colazzo, P. Stefanelli, Semi-braces and the Yang-Baxter equation, J. Algebra 483 (2017) $163-187$. URL https://doi.org/10.1016/j.jalgebra.2017.03.035

[14] F. Catino, I. Colazzo, P. Stefanelli, Skew left braces with non-trivial annihilator, J. Algebra Appl. 18 (2) (2019) $1950033,23$.

URL https://doi.org/10.1142/S0219498819500336 
[15] F. Catino, I. Colazzo, P. Stefanelli, The matched product of set-theoretical solutions of the Yang-Baxter equation, J. Pure Appl. Algebra 224 (3) (2020) 1173-1194.

URL https://doi.org/10.1016/j·jpaa.2019.07.012

[16] F. Catino, I. Colazzo, P. Stefanelli, The Matched Product of the Solutions to the Yang-Baxter Equation of Finite Order, Mediterr. J. Math. 17, 58 (2020).

URL https://doi org/10.1007/s00009-020-1483-y

[17] F. Catino, I. Colazzo, P. Stefanelli, Set-theoretic solutions to the Yang-Baxter equation and generalized semibraces, arxiv preprint arXiv:2004.01606.

URL https://arxiv.org/abs/2004.01606

[18] F. Catino, M. Mazzotta, P. Stefanelli, Set-theoretical solutions of the Yang-Baxter and pentagon equations on semigroups, Semigroup Forum 100 (3) (2020) 1-26.

URL https://doi.org/10.1007/s00233-020-10100-x

[19] F. Cedó, Left braces: solutions of the Yang-Baxter equation, Adv. Group Theory Appl. 5 (2018) 33-90. URL https://doi .org/10.4399/97888255161422

[20] F. Cedó, E. Jespers, J. Okniński, An abundance of simple left braces with abelian multiplicative Sylow subgroups, Rev. Mat. Iberoam.

URL https://www.ems-ph.org/journals/of_article.php?jrn=RMI\&doi=1168

[21] F. Cedó, E. Jespers, J. Okniński, Braces and the Yang-Baxter equation, Comm. Math. Phys. 327 (1) (2014) 101116.

URL https://doi .org/10.1007/s00220-014-1935-y

[22] F. Cedó, E. Jespers, J. Okniński, Every finite abelian group is a subgroup of the additive group of a finite simple left brace, J. Pure Appl. Algebra 225 (1) (2021) 106476.

URL http://www.sciencedirect.com/science/article/pii/S0022404920301778

[23] F. Cedó, E. Jespers, C. Verwimp, Structure monoids of set-theoretic solutions of the Yang-Baxter equation, arxiv preprint arXiv:1912.09710.

URL https://arxiv.org/abs/1912.09710

[24] F. Cedó, A. Smoktunowicz, L. Vendramin, Skew left braces of nilpotent type, Proc. Lond. Math. Soc. (3) 118 (6) (2019) 1367-1392.

URL https://doi.org/10.1112/plms.12209

[25] A. H. Clifford, G. B. Preston, The algebraic theory of semigroups. Vol. I, Mathematical Surveys, No. 7, American Mathematical Society, Providence, R.I., 1961.

[26] K. Cvetko-Vah, C. Verwimp, Skew lattices and set-theoretic solutions of the Yang-Baxter equation, J. Algebra 542 (2020) 65-92.

URL https://doi.org/10.1016/j.jalgebra.2019.10.007 
[27] V. G. Drinfel' d, On some unsolved problems in quantum group theory, in: Quantum groups (Leningrad, 1990), vol. 1510 of Lecture Notes in Math., Springer, Berlin, 1992, pp. 1-8.

URL https://doi.org/10.1007/BFb0101175

[28] P. Etingof, T. Schedler, A. Soloviev, Set-theoretical solutions to the quantum Yang-Baxter equation, Duke Math. J. 100 (2) (1999) 169-209.

URL http://dx.doi .org/10.1215/S0012-7094-99-10007-X

[29] T. Gateva-Ivanova, S. Majid, Matched pairs approach to set theoretic solutions of the Yang-Baxter equation, J. Algebra 319 (4) (2008) 1462-1529.

URL https://doi.org/10.1016/j.jalgebra.2007.10.035

[30] T. Gateva-Ivanova, M. Van den Bergh, Semigroups of I-type, J. Algebra 206 (1) (1998) 97-112.

URL http://dx.doi.org/10.1006/jabr.1997.7399

[31] L. Guarnieri, L. Vendramin, Skew braces and the Yang-Baxter equation, Math. Comp. 86 (307) (2017) 25192534.

URL https://doi.org/10.1090/mcom/3161

[32] M. Hall, Jr., The theory of groups, The Macmillan Co., New York, N.Y., 1959.

[33] J. M. Howie, Fundamentals of semigroup theory, vol. 12 of London Mathematical Society Monographs. New Series, The Clarendon Press, Oxford University Press, New York, 1995, oxford Science Publications.

[34] E. Jespers, L. Kubat, A. Van Antwerpen, L. Vendramin, Factorizations of skew braces, Math. Ann. 375 (3-4) (2019) 1649-1663.

URL https://doi.org/10.1007/s00208-019-01909-1

[35] E. Jespers, A. Van Antwerpen, Left semi-braces and solutions of the Yang-Baxter equation, Forum Math. 31 (1) (2019) 241-263.

URL https://doi .org/10.1515/forum-2018-0059

[36] M. Kunze, Zappa products, Acta Math. Hungar. 41 (3-4) (1983) 225-239.

URL https://doi.org/10.1007/BF01961311

[37] V. Lebed, Cohomology of idempotent braidings with applications to factorizable monoids, Internat. J. Algebra Comput. 27 (4) (2017) 421-454.

URL https : //doi.org/10.1142/S0218196717500229

[38] J.-H. Lu, M. Yan, Y.-C. Zhu, On the set-theoretical Yang-Baxter equation, Duke Math. J. 104 (1) (2000) 1-18. URL http://dx.doi .org/10.1215/S0012-7094-00-10411-5

[39] M. E. Malandro, Enumeration of finite inverse semigroups, Semigroup Forum 99 (3) (2019) 679-723.

URL https://doi .org/10.1007/s00233-019-10054-9 
[40] D. K. Matsumoto, K. Shimizu, Quiver-theoretical approach to dynamical Yang-Baxter maps, J. Algebra 507 (2018) 47-80.

URL https://doi.org/10.1016/j.jalgebra.2018.04.003

[41] K. Nejabati Zenouz, Skew braces and Hopf-Galois structures of Heisenberg type, J. Algebra 524 (2019) 187225.

URL https://doi.org/10.1016/j.jalgebra.2019.01.012

[42] W. R. Nico, On the regularity of semidirect products, J. Algebra 80 (1) (1983) 29-36.

URL https : //doi .org/10.1016/0021-8693(83)90015-7

[43] M. Petrich, Inverse semigroups, Pure and Applied Mathematics (New York), John Wiley \& Sons, Inc., New York, 1984, a Wiley-Interscience Publication.

[44] M. Petrich, N. R. Reilly, Completely regular semigroups, vol. 23 of Canadian Mathematical Society Series of Monographs and Advanced Texts, John Wiley \& Sons, Inc., New York, 1999, a Wiley-Interscience Publication.

[45] G. B. Preston, Semidirect products of semigroups, Proc. Roy. Soc. Edinburgh Sect. A 102 (1-2) (1986) $91-102$. URL https://doi.org/10.1017/S0308210500014505

[46] W. Rump, A decomposition theorem for square-free unitary solutions of the quantum Yang-Baxter equation, Adv. Math. 193 (1) (2005) 40-55.

URL https://doi.org/10.1016/j.aim.2004.03.019

[47] W. Rump, Braces, radical rings, and the quantum Yang-Baxter equation, J. Algebra 307 (1) (2007) $153-170$. URL https://doi.org/10.1016/j.jalgebra.2006.03.040

[48] W. Rump, Quasi-linear cycle sets and the retraction problem for set-theoretic solutions of the quantum YangBaxter equation, Algebra Colloq. 23 (1) (2016) 149-166.

URL https://doi.org/10.1142/S1005386716000183

[49] W. Rump, Set-theoretic solutions to the Yang-Baxter equation, skew-braces, and related near-rings, J. Algebra Appl. 18 (8) (2019) 1950145, 22.

URL https://doi.org/10.1142/S0219498819501457

[50] W. Rump, Affine structures of decomposable solvable groups, J. Algebra 556 (2020) 725-749.

URL https://doi.org/10.1016/j.jalgebra.2020.04.004

[51] W. Rump, Classification of indecomposable involutive set-theoretic solutions to the Yang-Baxter equation, Forum Math. 32 (4) (2020) 891-903.

URL https://doi .org/10.1515/forum-2019-0274

[52] A. Smoktunowicz, L. Vendramin, On skew braces (with an appendix by N. Byott and L. Vendramin), J. Comb. Algebra 2 (1) (2018) 47-86.

URL https://doi .org/10.4171/JCA/2-1-3 
[53] A. Soloviev, Non-unitary set-theoretical solutions to the quantum Yang-Baxter equation, Math. Res. Lett. 7 (5-6) (2000) 577-596.

URL http://dx.doi.org/10.4310/MRL.2000.v7.n5.a4

[54] D. Stanovskỳ, P. Vojtěchovskỳ, Idempotent solutions of the Yang-Baxter equation and twisted group division, arXiv preprint arXiv:2002.02854.

URL https: //arxiv.org/abs/2002.02854

[55] L. Vendramin, Extensions of set-theoretic solutions of the Yang-Baxter equation and a conjecture of GatevaIvanova, J. Pure Appl. Algebra 220 (5) (2016) 2064-2076.

URL https://doi.org/10.1016/j.jpaa.2015.10.018

[56] S. Wazzan, Zappa-Szép products of semigroups, Applied Mathematics 6 (06) (2015) 1047.

URL http://dx.doi.org/10.4236/am.2015.66096

[57] C. N. Yang, Some exact results for the many-body problem in one dimension with repulsive delta-function interaction, Phys. Rev. Lett. 19 (1967) 1312-1315.

URL https://doi.org/10.1103/PhysRevLett.19.1312

[58] D. Yang, The interplay between $k$-graphs and the Yang-Baxter equation, J. Algebra 451 (2016) 494-525.

URL https://doi.org/10.1016/j·jalgebra.2016.01.001 